\title{
CLASIFICACIÓN TRÓFICA Y CINÉTICA TEMPORAL DE LAS RELACIONES ENTRE PRODUCCIÓN Y CONSUMO EN LA CAPA FÓTICA DE LA PRESA DE UN EMBALSE ECUATORIAL COLOMBIANO
}

\author{
John Jairo Ramírez-Restrepo ${ }^{1 *}$, Jonathan Johnson ${ }^{1}$ \& Rigoberto Vahos ${ }^{1}$ \\ ${ }^{1}$ Universidad de Antioquia, Instituto de Biología, Grupo de Limnología Básica y Experimental, apartado 1226, \\ Medellín, Colombia. \\ E-mails: johnra77@gmail.com (*autor correspondiente); balinor48@yahoo.com; rivahos@hotmail.com
}

\begin{abstract}
Resumen: Se estudió la clasificación trófica y la cinética temporal de las razones P/B, P/R, PPN/PPB y R/B en la zona de represa del embalse Riogrande II $\left(06^{\circ} 35^{\prime}-06^{\circ} 28^{\prime} \mathrm{N}, 75^{\circ} 32^{\prime}-75^{\circ} 25^{\prime} \mathrm{O}\right)$. Se midieron la producción primaria bruta $(\mathrm{PPB})$ y la neta $(\mathrm{PPN})$ y la respiración $(\mathrm{R})$ en cinco profundidades dentro de la zona fótica. Los muestreos se realizaron cada mes, entre julio 2002 y junio 2003. La biomasa fitoplantónica (B) se cuantificó en muestras integradas dentro de la zona fótica. A partir de los valores máximos de los perfiles de PPB y R se establecieron los coeficientes fotosintéticos (PPBmáx/B) y metabólico (Rmáx/B), y la razón PPBmáx/Rmáx. La razón entre los valores medios de PPN y PPB muestra que PPN fue 5,2 veces PPB, lo que denota mayor R. Estas consideraciones implican que: 1) la estructura de biomasa presente se mantiene dada la neguentropía relativamente alta; y 2) metabólicamente, el sistema en la zona de represa es más autotrófico que heterotrófico, comportándose como un sumidero de carbono; por ello, parte de la biomasa no se respira, lo que implica que hay PPN disponible en el embalse. El coeficiente metabólico osciló alrededor de la unidad, confirmando la relativa estabilidad del ensamblaje fitoplanctónico, con algunos periodos catabólicos, pero primando los de carácter anabólico, lo que efectivamente convierte el sistema en un sumidero de Carbono capaz de mantenerse en condición estable y resistente a los aportes entrópicos. La razón $\mathrm{P} / \mathrm{R}$, con un valor medio de 1,61, muestra claramente un desbalance entre el material producido y el eliminado o consumido por R. Considerando la concentración de B fitoplanctónica (17,1 \pm 11,7 mgCl.a.m-3), la represa del cuerpo de agua investigado es eutrófica. Los valores medios del coeficiente de asimilación confirman esta condición.
\end{abstract}

Palabras clave: Colombia; metabolismo trófico; relación P/B; relación P/R; relación R/B.

TROPHIC CLASSIFICATION AND TEMPORAL KINETICS OF PRODUCTION AND CONUMPTION IN THE PHOTIC LAYER OF THE DAM ZONE IN AN EQUATORIAL COLOMBIAN RESERVOIR. We have studied the kinetic temporal and trophic classification of P/B, P/R, NPP/GPP, and R/B ratios in the dam zone of Riogrande II reservoir II $\left(06^{\circ} 35^{\prime}-06^{\circ} 28^{\prime} \mathrm{N}, 75^{\circ} 32^{\prime}-75^{\circ} 25^{\prime} \mathrm{W}\right)$. We measured gross (GPP) and net (NPP) primary production and respiration (R) at five depths within the photic zone. Samples were taken every month between July 2002 and June 2003. Phytoplankton biomass (B) was quantified in samples integrated within the photic zone. From the maximum values of GPP and R profiles, photosynthetic coefficient (GPPmax/B), GPPmax/Rmax ratio and metabolic coefficient (Rmax/B) were established. The ratio between the mean values of NPP and GPP shows that NPP was 5.2 times GPP, reflecting higher R. These considerations imply that: 1 ) the structure of biomass present remains due to the neguentropy relatively high; and 2) metabolically, the system in the dam zone is more autotrophic than heterotrophic, behaving as a carbon sink; therefore, part of the biomass is not breathed implying that there is available PPN. The 
metabolic coefficient ranged around the unit, confirming the relative stability of the phytoplankton assemblage, with some catabolic periods, but prioritizing the anabolic character, which effectively present the system as a carbon sink capable to maintain a stable condition and resistant to entropic contributions. $\mathrm{P} / \mathrm{R}$ ratio, with an average value of 1.61 , shows clearly an imbalance between the produced material and the removed or consumed by R. Considering the concentration of phytoplankton biomass $(17.1 \pm 11.7$ mgCl.a.m-3), the dam zone of the investigated water body is eutrophic. The mean value of photosynthetic coefficient confirms this condition.

Keywords: Colombia; $\mathrm{P} / \mathrm{B}$ ratio; $\mathrm{P} / \mathrm{R}$ ratio; $\mathrm{R} / \mathrm{B}$ ratio; trophic metabolism

\section{INTRODUCCIÓN}

Los ecosistemas están animados por un suplemento de energía que varía en un amplio espectro de escalas temporales y en el que toda la eficiencia trófica, depende (en última instancia) de la eficacia del primer paso: la cosecha y conversión de luz solar "contenida" en los enlaces de compuestos químicos intermedios de alta energía (Vincent 1990). Este proceso, denominado producción primaria o producción básica $(\mathrm{P})$, se define como la velocidad a la que es almacenada la energía por la actividad fotosintética o quimiosintética de organismos productores-algas en este caso-en forma de sustancias orgánicas susceptibles de ser utilizadas como material alimenticio que fluye a través de las redes tróficas (Odum 1972). Puede entenderse también como el peso del nuevo material orgánico o biomasa que se forma en un periodo determinado (Esteves 1998). Estas definiciones dan pie para concebir a la $\mathrm{P}$ como un proceso termodinámico generador de orden, ganado bajo la forma de material orgánico y representado en una estructura de biomasa (B) o nuevo protoplasma.

Una consecuencia de la segunda ley de la termodinámica, es que las estructuras ganadas mediante un proceso tienden a degradarse y a desaparecer en otro; en otras palabras, el orden se convierte en desorden. Debe asumirse entonces a $\mathrm{P}$ como una cara de la moneda del metabolismo autotrófico-heterotrófico del sistema que describe sólo la parte anabólica del mismo. La otra cara del mismo es descrita por la respiración (R) - llevada a cabo por varios tipos de organismos y cuya suma equivale a la respiración de la comunidad, $\mathrm{R}_{\mathrm{com}}=$ $\mathrm{R}_{\text {autotrofos }}+\mathrm{R}_{\text {heterótrofos }}-\mathrm{y}$ que para Odum (1972) y
Westlake et al. (1980) ha de encargarse de "eliminar el desorden" y bombearlo en forma de nutrientes inorgánicos, $\mathrm{CO}_{2}$ y otros gases. La $\mathrm{R}$ sería, pues, un proceso neguentrópico representante de la parte catabólica del metabolismo autotrófico-heterotrófico. Por ello, Reynolds (2006) expresa que los sistemas biológicos re-enrutan parte del flujo disipativo entrópico (dS) en el mantenimiento (R) de $\mathrm{B}$ o materia orgánica construida o elaborada mediante la $\mathrm{P}$. La mayor parte de la energía potencial en $\mathrm{P}$ se disipará como calor mediante R. Esta relación entre el flujo de orden y el flujo disipativo, "entropía negativa" o neguentropía, constituye el fundamento de la "Paradoja de Schrödinger" referida a que los sistemas biológicos deben bombear continuamente desorden para mantener así el orden ganado. El estado estable del sistema se alcanzará cuando las entradas (P/orden) compensen las salidas de material y energía (R/Neguentropía) para disipar el desorden.

Odum (1972) considera a la producción primaria bruta (PPB) como una "velocidad total" de fotosíntesis, incluida la materia orgánica usada en la respiración "durante el periodo de medición" y la designa también como fotosíntesis total o asimilación total; podría decirse que es la suma del proceso anabólico $(\mathrm{P})$ y el catabólico $(\mathrm{R})$ del metabolismo. Por su parte, la asimilación o producción primaria neta (PPN) de la comunidad es la proporción de la materia orgánica almacenada que no ha sido utilizada por los heterótrofos (PPN = PPB - R) "durante el periodo considerado", posiblemente equiparable al orden que aún permanece en el sistema; por ello, ha de tenerse en cuenta que aumentos de PPN implican reservas de carbono no usadas por el mismo. Los valores 
negativos que pueden presentarse en la PPN se deben a los acrecimos de $\mathrm{R}$ para eliminar el desorden presente en un momento dado, garantizando así el mantenimiento del sistema en un estado estable. Las disminuciones de R implicarán entonces valores positivos de PP, carbono disponible en el sistema e incrementos de la B de los productores primarios.

Fundamentados en las relaciones anabolismocatabolismo del metabolismo autotróficoheterotrófico, algunos autores proponen varios índices como estimadores de la estabilidad de un sistema. La primera de ellas es la denominada razón P/B entendida por Margalef $(1974,1983)$ como "eficiencia fotosintética" y por Westlake et al. (1980) y otros autores como un "coeficiente o número de asimilación fotosintética" calculado a partir de la razón entre una variable de entrada (P) y otra de salida (B). Esta razón enuncia más que $\mathrm{P}$ por sí sola, pues es una tasa que involucra implícitamente a $\mathrm{P}$, a B y a R. Por ello, puede decirse que es una expresión que muestra cuánta ganancia de orden se produce mediante fotosíntesis-producción a partir de un ensamblaje algal presente y ordenado que asimila, en mayor o menor grado, el flujo de energía radiante, pero que puede llegar a sucumbir bajo su propio sombreo ("algal self-shade"). Valores de la razón P/B cercanos a uno representan un balance entre entradas (P) y salidas (B), es decir, el logro de un estado estable para la comunidad, ensamblaje o sistema tratado; los valores mayores o inferiores a la unidad significarán desviaciones respecto a dicho estado. Igual sucede con las razones $\mathrm{PPN} / \mathrm{PPB}, \mathrm{P} / \mathrm{R}$ y R/B. Esta última, conocida como "coeficiente metabólico" o razón de Schrödinger (Odum 1972), establece una relación entre el desorden, eliminado por la R (neguentropía), y el orden, representado en $\mathrm{B}$ y generado mediante $\mathrm{P}$. El aumento de la razón R/B muestra la capacidad del sistema para quemar energía ( $R$ es costo de manutención), y por tanto, su aptitud para soportar disturbios provenientes de los alrededores (Angelini \& Gomes 2008). Además, si la razón $\mathrm{R} / \mathrm{B}>1$, el sistema tiene un metabolismo heterotrófico regido catabólicamente; cuando $\mathrm{R}<$ 1 , el sistema es autotrófico y se rige por el anabolismo. Relacionada con la expresión anterior, se encuentra la razón P/R (Odum 1972): si R < P, el sistema es autotrófico y se comporta como un sumidero de Carbono; si $\mathrm{R}>\mathrm{P}$, el sistema es heterotrófico y es además una fuente de $\mathrm{CO}_{2}$.

En Colombia, unos pocos estudios han abordado el tema de la PP en embalses. Naundorf (1990) en el embalse la Salvajina, un sistema oligotrófico, encontró valores medios 170,7 mgC. $m^{-3} \cdot h^{-1}$ para la PPB; Gaviria (1991), en el embalse La Regadera, un cuerpo de agua medianamente eutrófico, halló valores de 112,0; y de 90,0 en el embalse Chisacá (aparentemente eutrófico); y Aguirre-Ramírez et al. (2007) estudiaron la $\mathrm{P}$ de dos columnas de agua del embalse El Peñol, encontrando valores bajos (18,8 mgC.m${ }^{3} \cdot \mathrm{h}^{-1}$ en la primera y 17,5 en la segunda). Estos trabajos, aunque pocos, parecen concordar con lo expresado por Roldán-Pérez \& Ramírez-Restrepo (2008) en cuanto a que en los embalses tropicales no se espera una PP alta debido a que esta depende principalmente del fitoplancton el cual presenta limitaciones de reproducción debidas a la pobreza de nutrientes en la superficie y a la alta turbidez generalizada que hace posible una transparencia de tan sólo dos a tres metros en promedio y, en muchos casos, menor un metro de profundidad.

En el embalse Riogrande II se han realizado investigaciones relacionadas con los componentes que afectan la penetración de luz en el embalse (Ramírez-Restrepo et al. 2015), la ecología de Ceratium furcoides (Gonyaulacales, Ceratiaceae; Bustamante Gil et al. 2012), la de cuatro especies de Staurastrum (Desmidiales, Desmidiaceae; Loaiza-Restano et al. 2011), las tasas de sedimentación en la represa del cuerpo de agua (Montoya \& Ramírez-Restrepo 2007), y finalmente, la caracterización limnológica general del embalse Mazo et al. (2015), el efecto de la posición de la compuerta en la estructura térmica (FrancoVelásquez et al. 2010), y la modelación de la estructura térmica y el patrón de corrientes (Franco-Velásquez 2011). Sin embargo, la $\mathrm{P}$ y los factores asociados a ella en dicho embalse, al igual que en muchos otros de la región, no ha sido abordada.

Existen en el embalse Riogrande II algunos factores que consideramos importantes para explicar la dinámica de la $\mathrm{P}$ en la escala temporal. En primera instancia, la morfología meándrica y la zona extensa de las "colas", representadas por los ríos Chico y Grande (Figura 1) pueden ejercer una 
especial influencia en la carga de nutrientes que llega a la zona de presa, pues, podrían ser retenidos en estas "colas", aumentando en la presa los niveles de transparencia, y mejorando el clima óptico del sitio, pero presentando una menor concentración de nutrientes. Un segundo factor a considerar es que en la cuenca de captación existen dos períodos lluviosos, abriljunio y septiembre-noviembre (Figura 2); quizás la alternancia de estos periodos influencie las variaciones en la dinámica del proceso evaluado, pues la lluvia arrastra gran cantidad de nutrientes por las vertientes hacia el embalse, lo que puede aumentar los niveles de $\mathrm{P}$ y alterar el balance entropía-neguentropía en el interior del mismo. Además, según Franco-Velásquez (2011) y Mazo et al. (2015) y, el embalse Riogrande II presenta una estratificación térmica permanente, un patrón atelomíctico de mezcla y unas profundidades fóticas y de mezcla que avanzan muy poco en la columna de agua, al igual que una temperatura mayor de $16^{\circ} \mathrm{C}$ en el hipolimnio. Esta última condición, de acuerdo con Esteves (1998), es típica de cuerpos de agua en regiones tropicales y puede ser causa de hipoxia/anoxia de fondo debido a las altas tasas respiratorias de la materia orgánica sedimentada, que ocasionan liberación de nutrientes y gases que no ascenderían hasta el epilimnio debido a la poca profundidad de la mezcla y a la barrera representada por la termoclina. En principio, las fuentes autóctonas, al igual que las alóctonas, no estarían disponibles en la represa para los procesos relacionados con $\mathrm{P}$ y $\mathrm{R}$.

Considerando entonces: 1) que en los embalses Considerando entonces: 1) que en los embalses tropicales no se espera una PP alta (Roldán-Pérez

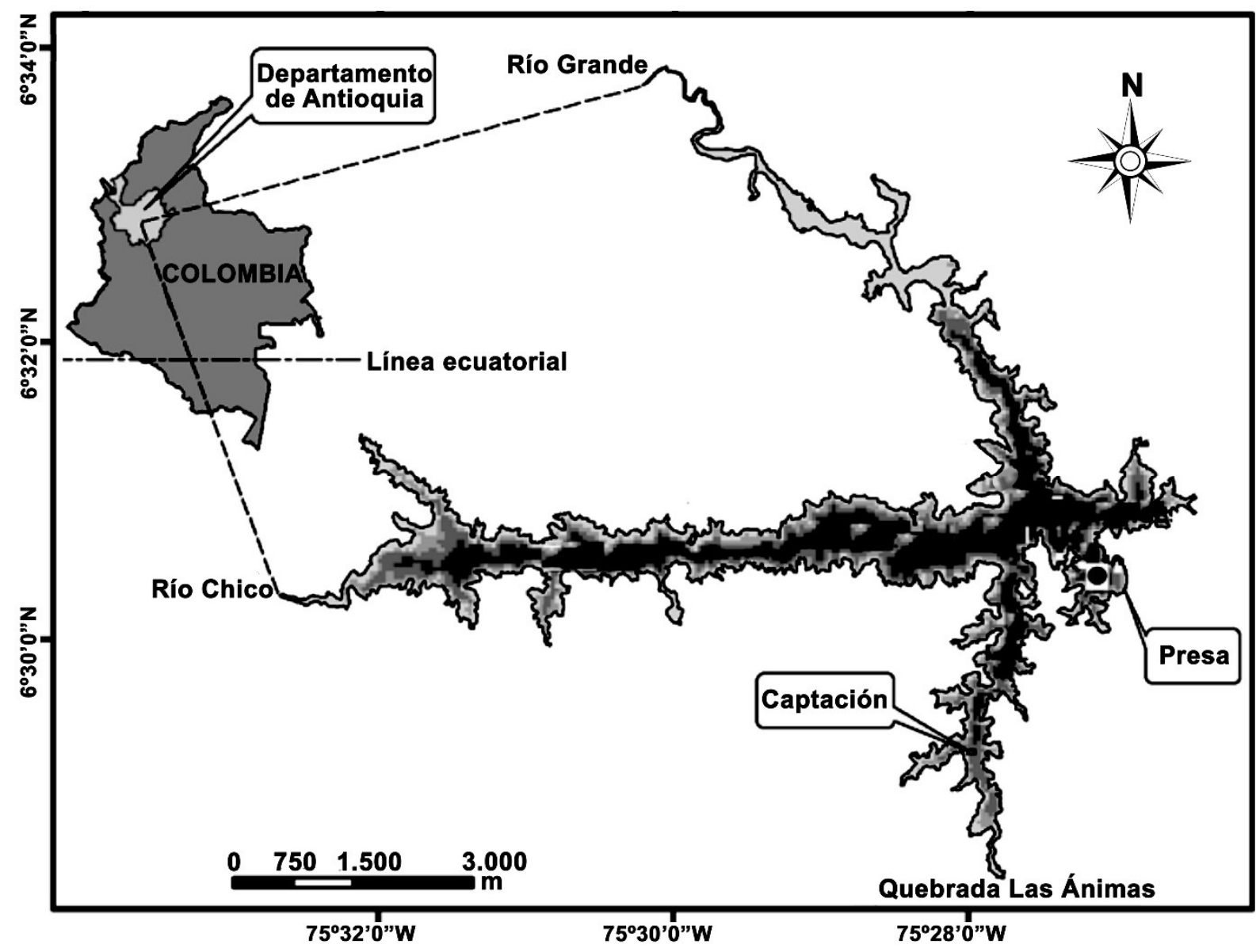

Figura 1. Mapa del embalse Riogrande II mostrando la ubicación de la estación de muestreo (Fuente: Franco-Velásquez et al. 2010).

Figure 1. Riogrande II reservoir's map showing the location of the sampling station (Font: Franco-Velásquez et al. 2010). 


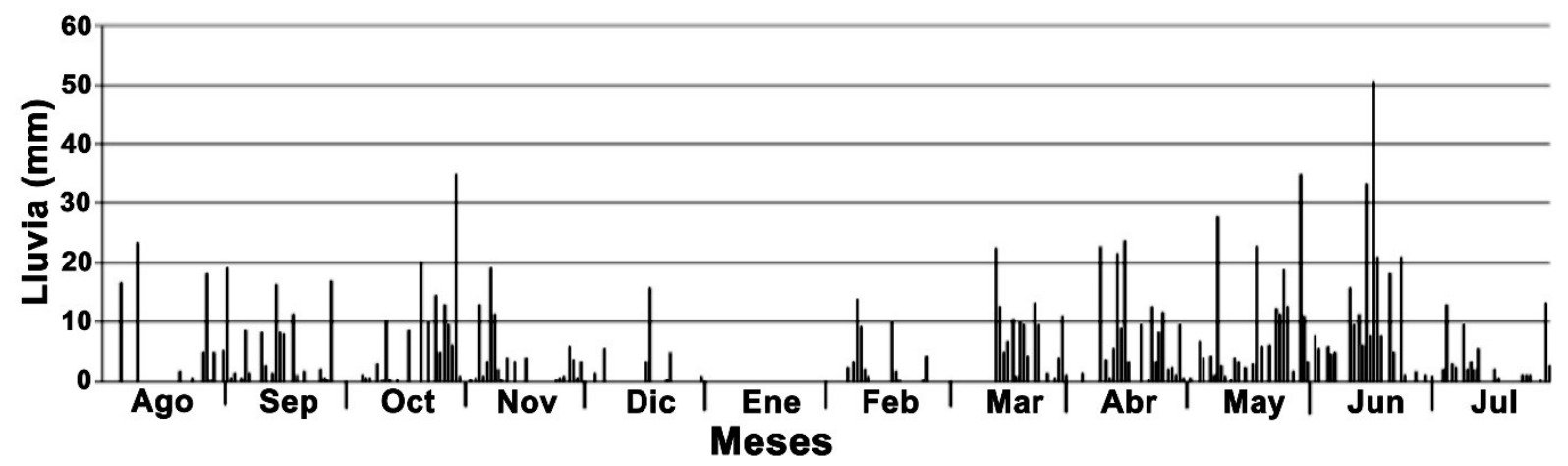

Figura 2. Embalse Riogrande II. Pluviosidad diaria total en el periodo agosto de 2002 - julio de 2003.

Figure 2. Riogrande II Reservoir. Total daily rainfall during the period August 2002 - July 2003

\& Ramírez-Restrepo 2008), 2) la posible influencia de la pluviosidad, 3) la desmejora del clima óptico en la zona de la presa, 4) la imposibilidad manifiesta de las fuentes alóctonas y autóctonas de material inorgánico disuelto de llegar hasta ella y de ascender desde el fondo hasta la zona fótica de la presa debido al patrón atelomíctico del cuerpo de agua, y 5) la poca profundidad de mezcla, nos preguntamos: 1) ¿Cómo es la cinética temporal de P, R y B?; 2) Cómo es en la zona de represa la homeostasis o estabilidad del sistema valorada mediante las razones $\mathrm{P} / \mathrm{B}, \mathrm{P} / \mathrm{R}, \mathrm{PPN} / \mathrm{PPB}$ y R/B en la escala temporal anual?; 3) ¿Cómo es su comportamiento metabólico?; 4) ¿Cuál es su clasificación trófica?; y, por último, 5) ¿qué factores limitan la P en ella?

Para estas cuestiones, proponemos las siguientes hipótesis: si la cinética de las variables mencionadas es altamente mudable en la escala temporal; y si está influenciada por el clima ambiental (temperatura y pluviosidad) y lumínico (razón profundidad eufótica-profundidad de mezcla; Deu/Dmix imperante en la presa; y si ni las fuentes alóctonas ni las autóctonas influencian esta zona del embalse, entonces (1) el fitoplancton de la zona investigada está limitado por nutrientes, (2) tiene un metabolismo de carácter heterotrófico, y (3) se comporta como un sistema proveedor de $\mathrm{CO}_{2}$ por presentar mayores tasas de $\mathrm{R}$ que de $\mathrm{P}$, razones $\mathrm{P} / \mathrm{B}$ y $\mathrm{R} / \mathrm{B}$ mayores que la unidad, $\mathrm{y}$ PPN/PPB menores que uno. Además, (4) en los periodos húmedos, cuando disminuyan el clima lumínico y las concentraciones de nutrientes, se presentan los mayores valores de P. Finalmente, (5) la zona de presa del cuerpo de agua está alejada la mayor parte del tiempo de una condición estable.

\section{MATERIAL Y MÉTODOS}

\section{Área de estudio}

El embalse Riogrande II se encuentra localizado en la parte central del departamento de Antioquia (Colombia), en la cuenca del río Grande, situada al norte de la ciudad de Medellín $\left(06^{\circ} 35^{\prime}-06^{\circ} 28^{\prime} \mathrm{N}\right.$, $75^{\circ} 32^{\prime}-75^{\circ} 25^{\prime} \mathrm{O}$; Franco-Velásquez 2010; Figura 1). Las temperaturas de la zona del embalse oscilan entre los $14^{\circ} \mathrm{C}$ y los $18^{\circ} \mathrm{C}$. Existen en ella dos períodos lluviosos, uno entre abril y junio y otro entre septiembre y noviembre, determinados principalmente por el desplazamiento de la Zona de Convergencia Intertropical. Las épocas de lluvias bajas (denominadas localmente épocas secas o de verano) van de diciembre a marzo y de julio a agosto, siendo la primera menos lluviosa que la segunda. Los mayores valores de humedad se presentan entre octubre y noviembre con promedios cercanos al $83 \%$.

La represa del embalse está ubicada a $1 \mathrm{~km}$ de la estructura en tierra y es la que presenta las mayores profundidades ( 35 a $40 \mathrm{~m}$ ). Esta estructura crea el embalse que almacena 253 millones de $\mathrm{m}^{3}$ de agua e inunda 1,100 ha de los municipios de San Pedro de los Milagros, Santa Rosa de Osos, Entrerríos, Donmatías y Belmira.

La torre de captación se localiza en un brazo del embalse que sube por la cuenca de la quebrada Las Ánimas. El agua se capta en la torre por un sistema de cuatro compuertas rectangulares distribuidas en dos niveles así: entre los niveles 2257.53 - $2254.03 \mathrm{~m}$ s.n.m. (compuerta superior) se capta un caudal máximo de $6,4 \mathrm{~m}^{3} / \mathrm{s}$ para la planta de tratamiento de aguas Manantiales. Entre 
los niveles 2249.47-2245.97 m s.n.m. (dos compuertas inferiores) se capta un caudal máximo de $40 \mathrm{~m}^{3} \cdot \mathrm{s}^{-1}$ para la central hidroeléctrica La Tasajera. Por la compuerta restante se capta en ocasiones para la planta manantiales (Franco-Velásquez et al. 2010).

Sus aguas, aunque turbias, poseen excelente calidad química (sólidos disueltos máx18 mg.1-1). Son aguas muy blandas ( $\left.5 \mathrm{mg} \mathrm{CaCO}_{3} \cdot \mathrm{l}^{-1}\right)$, con altas concentraciones de hierro. Su tiempo medio de retención es de 72,8 días lo que lo categoriza como un embalse de la clase B o embalse de retención intermedia (EPM 1994, Porras-Zapata et al. 1997, Straškraba 1999).

Durante el periodo de muestreo el embalse presentó valores medios altos de clorofila $a$ activa, fósforo soluble reactivo, nitratos; y bajos del coeficiente de atenuación y transparencia, así como perfiles verticales de conductividad y $\mathrm{CO}_{2}$ total con incrementos hacia el fondo, $\mathrm{y}$ disminuciones de $\mathrm{pH}$ y oxígeno hacia esta profundidad en las cinco estaciones muestreadas (Mazo et al. 2015).

Franco-Velásquez (2011) determinó que el caudal de río Grande es, en promedio, tres veces mayor al del río Chico; por ello, río Grande al entrar al cuerpo de agua reparte su volumen entre el brazo del río Chico y el de la quebrada Las Animas. Las aguas de río Grande se internan en el río Chico hasta localizarse a $4 \mathrm{~km}$ de la cola de este río, aumentando su tiempo de residencia. Sobre el brazo de la quebrada Las Animas, el río Grande viaja directamente hasta la torre de captación a nivel de la compuerta superior. Por su parte, el río Chico ingresa al embalse como una corriente de fondo difícil de rastrear pues se mezcla rápidamente con el agua del embalse. En las proximidades de la torre de captación, dicha pluma se direcciona hacia la compuerta inferior debido al caudal extraído para generación de energía en la central Tasajera.

El embalse permanece estratificado a lo largo del año. Las variaciones de temperatura en el ciclo anual en la capa superficial oscilan entre 19,5$23,7^{\circ} \mathrm{C}$ (media: $21,6 \pm 1,35^{\circ} \mathrm{C}$ ). Esta capa superficial presenta variaciones en el espesor que están entre 3,0 - 6,0 $\mathrm{m}$ de profundidad. La zona del hipolimnio se caracteriza por presentar pocas variaciones a lo largo del año y su temperatura oscila entre los $17,4-18,2^{\circ} \mathrm{C}$ (media: $17,4 \pm 0,92$ ). Esta estructura térmica está fuertemente asociada a la dinámica de la corriente de densidad de río Grande que entra al cuerpo de agua como una corriente intrusiva a una profundidad de 5-10 m, modificando el decaimiento exponencial de la temperatura en la columna de agua (FrancoVelásquez 2011).

\section{Diseño de la investigación}

Entre agosto de 2002 y julio de 2003 se realizaron en la zona de represa 35 muestreos de variables limnológicas que incluyeron profundidad, transparencia, temperatura del aire, oxígeno disuelto, porcentaje de saturación de oxígeno, temperatura del agua, conductividad eléctrica, $\mathrm{pH}$, alcalinidad, turbiedad y fitoplancton, entre otras.

\section{Métodos para la medición de las variables físicas, químicas y biológicas}

Entre agosto de 2002 y julio de 2003 se realizaron en la zona de represa 35 muestreos de variables limnológicas que incluyeron profundidad, transparencia, temperatura del aire, oxígeno disuelto, porcentaje de saturación de oxígeno, temperatura del agua, conductividad eléctrica, $\mathrm{pH}$, alcalinidad, turbiedad y fitoplancton, entre otras (Tabla 1).

Los datos de pluviosidad se obtuvieron de Empresas públicas de Medellín. Los perfiles de temperatura y oxígeno disuelto en la columna de agua se llevaron a cabo a cada $0,25 \mathrm{~m}$ en los primeros diez metros. A partir de allí se realizaron a cada metro hasta el fondo. Las muestras de agua para medir $\mathrm{CO}_{2}$ total, $\mathrm{CO}_{2}$ libre, $\mathrm{HCO}_{3}{ }^{-}$, y $\mathrm{CO}_{3}=$, turbidez, conductividad eléctrica, alcalinidad y $\mathrm{pH}$ se extrajeron a cada cinco metros. Las concentraciones de sólidos suspendidos totales, fósforo soluble reactivo, nitrógeno total, amonio y nitrato en la zona fótica fueron estimadas mensualmente.

Para estimar PPB, PPN y R se pusieron dos botellas claras y dos oscuras en subsuperficie (asumida como $100 \% \mathrm{I}_{0}{ }^{\prime}$ ), 50\% $\mathrm{I}_{0}{ }^{\prime}, 25 \% \mathrm{I}_{0}{ }^{\prime}, 10 \% \mathrm{I} \mathrm{I}_{0} \mathrm{y}$ $1 \% \mathrm{I}_{0}$ ' (límite de la zona fótica). Las profundidades elegidas variaron en cada muestreo y se establecieron con base en un perfil lumínico efectuado con un cuantómetro LICOR. El factor usado para la conversión de los miligramos de oxígeno a miligramos de Carbono fue 0,312 (Cole 1983).

A partir de los valores máximos de los perfiles de PPB y $R$ ( $\mathrm{PPB}_{\text {máx y }} \mathrm{R}_{\text {máx }}$ ) en cada muestreo se 
Tabla 1. Variables climáticas, físicas, químicas y biológicas consideradas en esta investigación y sus respectivas unidades, materiales y/o método de medición.

Table 1. Climatic, physical, chemical and biological variables considered in this investigation and their respective units, materials and / or measurement method.

\begin{tabular}{|c|c|c|c|c|}
\hline Tipo & Variable & Unidad & Material/Método & Convención \\
\hline \multirow[t]{3}{*}{ Climáticas } & Pluviosidad & mm.mes ${ }^{-1}$ & Pluviómetro & Pl. \\
\hline & Velocidad del viento & $\mathrm{m} \cdot \mathrm{s}^{-1}$ & Anemómetro & $\mathrm{U}$ \\
\hline & Dirección del viento & Grados sexagesimales & Veleta de tela & $\mathrm{U}_{\mathrm{d}}$ \\
\hline \multirow[t]{6}{*}{ Físicas } & Transparencia & $\mathrm{m}$ & Disco de Secchi $(\varphi=0,20 \mathrm{~m})$ & $\mathrm{D}_{\mathrm{eu}}$ \\
\hline & Turbidez & NTU & Turbidímetro & $\mathrm{T}$ \\
\hline & Atenuación de luz & Ln (Unidades de luz).m-1 & Datalogger Licor & K \\
\hline & Sólidos suspendidos totales & mg..$^{-1}$ & Gravimetría & SST \\
\hline & Conductividad eléctrica & $\mu \mathrm{S} . \mathrm{cm}^{-1}$ & Conductímetro YSI & $\mathrm{C}_{25^{\circ}}$ \\
\hline & Perfil térmico & ${ }^{\circ} \mathrm{C}$ & Termistor-oxímetro YSI & $\Theta$ \\
\hline \multirow[t]{11}{*}{ Químicas } & Perfil de oxígeno disuelto & $\mathrm{mgO}_{2} \cdot \mathrm{l}^{-1}$ & Termistor-oxímetro YSI & O.D. \\
\hline & $\mathrm{pH}$ & Adimensional & Peachímetro Schöt Gerate & $\mathrm{pH}$ \\
\hline & $\mathrm{CO}_{2}$ total & mg.l-1 & Mackereth et al. (1978) & $\sum \mathrm{CO}_{2}$ \\
\hline & Dióxido de carbono libre & mg.l-1 & Mackereth et al. (1978) & $\mathrm{CO}_{2}$ \\
\hline & Bicarbonatos & mg..$^{-1}$ & Mackereth et al. (1978) & $\mathrm{HCO}_{3}^{-}$ \\
\hline & Carbonatos & mg.l $1^{-1}$ & Mackereth et al. (1978) & $\mathrm{CO}_{3}^{-2}$ \\
\hline & Alcalinidad total & $\mathrm{mgCaCO}_{3} \cdot \mathrm{l}^{-1}$ & Titulometría & A.T. \\
\hline & Nitrógeno total & $\mathrm{mgNO}_{3} \cdot \mathrm{l}^{-1}$ & Kjeldal & NTK \\
\hline & Amonio & $\mathrm{mgN}-\mathrm{NH}_{4} \cdot \mathrm{l}^{-1}$ & Nessler & $\mathrm{N}-\mathrm{NH}_{4}{ }^{+}$ \\
\hline & Nitratos & $\mathrm{mgN}-\mathrm{NO}_{3} \cdot \mathrm{l}^{-1}$ & Reducción Cadmio-Cobre & $\mathrm{N}-\mathrm{NO}_{3}^{-}$ \\
\hline & Fósforo soluble & $\mathrm{mgP}-\mathrm{PO}_{4} \cdot \mathrm{l}^{-1}$ & Ácido ascórbico & $\mathrm{P}-\mathrm{PO}_{4}{ }^{-3}$ \\
\hline \multirow[t]{8}{*}{ Biológicos } & Clorofila a (B) & mgCl.a.m ${ }^{-3}=$ mgCl. a..$^{-1}$ & Sartory \& Grobbelaar (1984) & B \\
\hline & B por unidad de zona fótica & mgCl. a.m-2 & $\begin{array}{l}\text { B } \times \text { profundidad media }(23 \\
\text { m). Kalff (1983) y Nixdorf \& } \\
\text { Deneke (1997) }\end{array}$ & $\sum \mathrm{B}$ \\
\hline & $\begin{array}{l}\text { Producción primaria bruta, } \\
\text { producción primaria neta y } \\
\text { respiración }\end{array}$ & $\operatorname{mgC} \cdot \mathrm{m}^{-3} \cdot \mathrm{h}^{-1}$ & Gaarder \& Gran (1927) & PPB, PPN, R \\
\hline & $\begin{array}{l}\text { PPB y R por unidad de área } \\
\text { de zona fótica }\end{array}$ & $\mathrm{mgC} \cdot \mathrm{m}^{-2} \cdot \mathrm{h}^{-1}$ & $\begin{array}{l}\text { Integración por regla del } \\
\text { trapecio }\end{array}$ & $\sum \mathrm{PPB}, \sum \mathrm{R}$ \\
\hline & $\begin{array}{l}\text { PPB y R en la zona fótica } x \\
\text { día }\end{array}$ & $\mathrm{mgC} \cdot \mathrm{m}^{-2} \cdot \mathrm{d}^{-1}$ & $\begin{array}{l}\text { Cálculo: } \sum \text { PPB } \times 12 \text { horas de } \\
\text { luz y } \sum \mathrm{R} \times 12 \text { horas de luz }\end{array}$ & $\sum \mathrm{PPB}_{\mathrm{d}}, \sum \mathrm{R}_{\mathrm{d}}$ \\
\hline & PPB anual & $\mathrm{gC} \cdot \mathrm{m}^{-2} \cdot \mathrm{a}^{-1}$ & $\begin{array}{l}\text { Integración por mes, regla } \\
\text { del trapecio }\end{array}$ & $\sum \sum \mathrm{PPB}$ \\
\hline & Razón o profundidad crítica & Adimensional & $\begin{array}{l}\sum_{1971)} \mathrm{PPB}_{\mathrm{d}} / \sum \mathrm{R}_{\mathrm{d}} \text { (Talling } 1965, \\
\end{array}$ & $\mathrm{q}_{\mathrm{c}}$ \\
\hline & Clima óptico & Adimensional & $\begin{array}{l}\text { Profundidad } \\
\text { fótica/profundidad de } \\
\text { mezcla y Profundidad fótica/ } \\
\text { profundidad afótica }\end{array}$ & $\begin{array}{l}\mathrm{D}_{\mathrm{eu}} / \mathrm{D}_{\mathrm{mix}} \\
\mathrm{D}_{\mathrm{eu}} / \mathrm{D}_{\mathrm{af}}\end{array}$ \\
\hline
\end{tabular}

establecieron el coeficiente fotosintético, coeficiente de asimilación o capacidad fotosintética $\left(\mathrm{PPB}_{\text {máx }} / \mathrm{B}\right)$, la razón de $\mathrm{PPB}_{\text {máx }} / \mathrm{R}_{\text {máx }}$ y el coeficiente metabólico $\left(\mathrm{R}_{\text {máx }} / \mathrm{B}\right)$ que, en este escrito, se denotarán en adelante como P/B, P/R y R/B.
Se determinó además la "razón o profundidad crítica” $\left(\mathrm{q}_{\mathrm{c}}\right)$ de Talling $(1965,1971)$ que permite establecer la profundidad de mezcla a la cual las pérdidas respiratorias (R) son balanceadas por las ganancias fotosintéticas (P). Si el valor de esta 
razón es uno, se está en el punto de compensación en donde PPB $\rightarrow$ R y PPN $\rightarrow 0$. Según Talling (1957) este valor puede también calcularse como 32R.

El valor del coeficiente de autosombreo algal (Ks) se calculó según Talling (1971) y Harrris et al. (1980) a partir de la relación entre el coeficiente de atenuación total $\left(\mathrm{K}_{\mathrm{T}}\right)$ y la concentración de Clorofila a (a). La pendiente de esta relación representa el valor de dicho coeficiente en $\mathrm{m}^{2}$ $(\mathrm{mgCl} . \mathrm{a})^{-1}$.

\section{Análisis estadístico}

El análisis estadístico efectuado es solo de carácter descriptivo y exploratorio. Para ello, se utilizaron gráficos y figuras pertinentes, y se calcularon los valores de la media aritmética, la desviación estándar y el coeficiente de variación de cada variable.

\section{RESULTADOS}

El régimen de pluviosidad fue altamente variable $(C V=184,3 \%)$. El periodo "seco" comprendió desde finales de diciembre hasta inicios de febrero, mientras que el lluvioso mostró dos cimas, una en octubre $(35,3 \mathrm{~mm})$ y otra en junio $(50,2 \mathrm{~mm})$ (Figura 2). La pluviosidad total anual fue de 1324,2 $\mathrm{mm}$. En los días de muestreo (Figura 2), la pluviosidad osciló entre $0 \mathrm{~mm}$, en enero 3 de 2003, y $8,2 \mathrm{~mm}$ en junio $1 \mathrm{del}$ mismo año. La variación fue de $63,8 \%$ y el promedio anual de $3,6 \mathrm{~mm}$. También se observaron dos picos de mayor pluviosidad dentro de la gráfica, lo que indica, tanto en el ciclo anual como en el diario, un comportamiento de carácter bimodal, consecuente con lo observado en algunas regiones ecuatoriales y tropicales.

La mayor variación la presentó la razón PPN/PPB debido a los valores negativos hallados (Tabla 2). Seis de las ocho razones exhibieron valores medios cercanos o inferiores a uno, y CV mayores que $20 \%$. Se observa además que tanto PPN como R, la razón PPN/PPB y q mostraron valores negativos (Tabla 2 ).

En la escala espacial vertical los valores máximos de PPB se presentaron entre los 0,25 y

Tabla 2. Dimensiones y valores de tendencia central y dispersión relativa de las variables relacionadas con la producción primaria en la represa del embalse Riogrande II ( $\mathrm{N}=12)$

Table 2. Dimensions and values of central tendency and dispersion relative variables related to primary production in the dam reservoir Riogrande II $(N=12)$.

\begin{tabular}{|c|c|c|c|c|c|}
\hline Variable & Dimensión & $V_{\text {mín }}$ & $\mathbf{V}_{\text {máx }}$ & & $\mathrm{CV}(\%)$ \\
\hline $\mathrm{D}_{\mathrm{eu}}$ & $\mathrm{m}$ & 3,34 & 7,37 & 5,02 & 28,2 \\
\hline $\mathrm{D}_{\text {mix }}$ & $\mathrm{m}$ & 2,67 & 8,33 & 4,89 & 58,0 \\
\hline $\mathrm{D}_{\text {máx }}$ & $\mathrm{m}$ & 22,30 & 41,50 & 35,17 & 17,6 \\
\hline $\mathrm{D}_{\text {eu }} / \mathrm{D}_{\text {máx }}$ & Adimensional & 0,09 & 0,33 & 0,15 & 47,8 \\
\hline $\mathrm{D}_{\mathrm{eu}} / \mathrm{D}_{\text {mix }}$ & Adimensional & 0,77 & 1,78 & 1,11 & 29,9 \\
\hline $\mathrm{D}_{\text {mix }} / \mathrm{D}_{\text {máx }}$ & Adimensional & 0,07 & 0,34 & 0,15 & 59,3 \\
\hline $\mathrm{D}_{\mathrm{eu}} / \mathrm{D}_{\mathrm{af}}$ & Adimensional & 0,10 & 0,49 & 0,19 & 61,6 \\
\hline$\sum \mathrm{PPB}$ & mgC.m $m^{-2} \cdot \mathrm{h}^{-1}$ & 6,20 & 244,1 & 104,7 & 66,2 \\
\hline $\mathrm{PPB}_{\text {máx }}$ & mgC. $\mathrm{m}^{-3} \cdot \mathrm{h}^{-1}$ & 43,4 & 124,8 & 75,8 & 27,4 \\
\hline $\mathrm{R}_{\text {máx }}$ & mgC. $\mathrm{m}^{-3} \cdot \mathrm{h}^{-1}$ & 26,0 & 165,4 & 61,1 & 63,8 \\
\hline $\mathrm{PPN}_{\text {máx }}$ & mgC. $\mathrm{m}^{-3} \cdot \mathrm{h}^{-1}$ & 87,4 & $-87,4$ & 14,7 & 294,7 \\
\hline $\mathrm{PPN} / \mathrm{PPB}$ & Adimensional & $-1,1$ & 0,7 & 0,2 & 354,1 \\
\hline$\sum \mathrm{R}$ & mgC. $\mathrm{m}^{-2} \cdot \mathrm{h}^{-1}$ & $-341,3$ & 483,4 & 116,3 & 172,1 \\
\hline B & mgCl.a.m & 1,9 & 41,5 & 17,1 & 68,7 \\
\hline$\sum \mathrm{B}$ & mgCl.a.m-2 & 44,6 & 953,6 & 392,5 & 68,7 \\
\hline$\Sigma \mathrm{B}, \Sigma \mathrm{R}$ & mgCl.a.m $\mathrm{m}^{-3}+$ mgC. $\mathrm{m}^{-3} \cdot \mathrm{h}^{-1}$ & 7,2 & 1073,2 & 508,8 & 55,7 \\
\hline$\sum \mathrm{PPB}_{\mathrm{d}}$ & mgC. $m^{-2} \cdot d^{-1}$ & 74,4 & 2929,2 & 1256,5 & 66,2 \\
\hline $\mathrm{PPB}_{\text {máx }} / \mathrm{R}_{\text {máx }}$ & Adimensional & 0,5 & 3,3 & 1,6 & 55,8 \\
\hline $\mathrm{PPB}_{\text {máx }} / \mathrm{B}$ & mgC. $(m g C l . a . h)^{-1}$ & 2,6 & 28,9 & 8,5 & 107,7 \\
\hline $\mathrm{R}_{\text {máx }} / \mathrm{B}$ & mgC.(mgCl.a.h) $)^{-1}$ & 0,9 & 38,6 & 8,6 & 136,2 \\
\hline $\mathrm{q}_{\mathrm{c}}$ & Adimensional & $-0,7$ & 2,3 & 0,9 & 104,1 \\
\hline$\sum \sum \mathrm{PPB}$ & $\mathrm{gC} \cdot \mathrm{m}^{-2} \cdot \mathrm{a}^{-1}$ & ---- & --- & 437,3 & ---- \\
\hline
\end{tabular}


2,19 m; en 11 de los 12 muestreos realizados, sólo uno se situó por debajo de esta profundidad llegando hasta los $6 \mathrm{~m}$. Los valores de $\mathrm{PPB}_{\text {máx }}$ oscilaron entre $43,4 \mathrm{mgC} \cdot \mathrm{m}^{-3} \cdot \mathrm{h}^{-1}$ en octubre 1 de 2002 y 124,8 en diciembre 2 de 2002 con un valor medio de 75,8 (Tabla 2). La $\sum \mathrm{PPB}_{\mathrm{d}}\left(\sum \mathrm{PPB} \times 12\right.$ horas de luz) varió considerablemente (CV $=66,2 \%)$ oscilando entre 74,4 y $2929,2 \mathrm{mgC} \cdot \mathrm{m}^{-2} \cdot \mathrm{d}^{-1}$ con un valor medio de 1256,5; la $\sum \sum$ PPB $\left(437,3 \mathrm{gC} . \mathrm{m}^{-2} \cdot \mathrm{a}^{-1}\right)$ fue bastante grande. $\mathrm{R}_{\text {máx }} \mathrm{y} \mathrm{PPB}_{\text {máx }}$ presentaron valores mayores que los de B (Figuras 3 y 4).

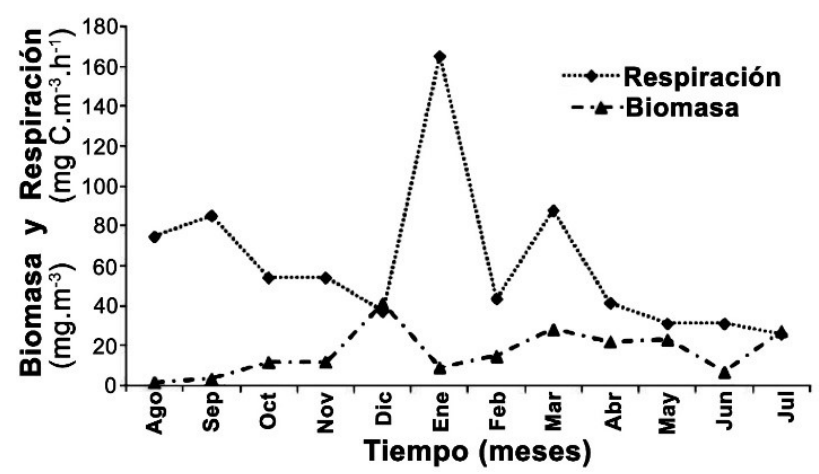

Figura 3. Embalse Riogrande II, Don Matías, Colombia. Variación temporal de la biomasa fitoplanctónica y la respiración.

Figure 3. Riogrande II Reservoir, Don Matias, Colombia. Temporal variation of phytoplankton biomass and respiration.

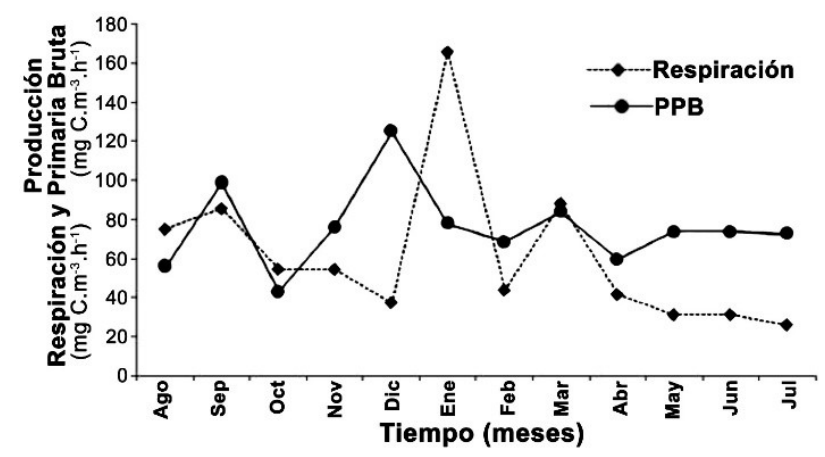

Figura 4. Embalse Riogrande II, Don Matías, Colombia. Variación temporal de la respiración y la producción primaria bruta.

Figure 4. Riogrande II Reservoir, Don Matias, Colombia. Temporal variation of respiration and gross primary production.

Por su parte, $\mathrm{R}_{\text {máx }}$ fue relativamente uniforme, excepto por los picos mostrados en septiembre, enero y marzo; además, fue mayor que $\mathrm{B}$ en 10 muestreos (Figura 3) y que $\mathrm{PPB}_{\text {máx }}$ sólo en enero; el resto del tiempo fue ligeramente inferior (Figura
4). B siempre fue mayor que $\mathrm{PPB}_{\text {máx }}$ (Figura 5). A su vez, la razón P/R (Figura 6) fue mayor que uno en ocho muestreos, con un valor medio de 1,6 y una variación temporal relativamente alta $(\mathrm{CV}=$ $55,8 \%)$.

El coeficiente de Asimilación fotosintética fue mayor que uno en 10 de las 12 colectas, su promedio fue de 8,5 \pm 9,2 $\mathrm{mgC}(\mathrm{mgCl} . \mathrm{a} . \mathrm{h})^{-1}$, con una pronunciada variación anual $(\mathrm{CV}=107,7 \%)$ (Figura 7). El comportamiento del mismo fue de tipo logarítmico $(\mathrm{p}<0,001)$.

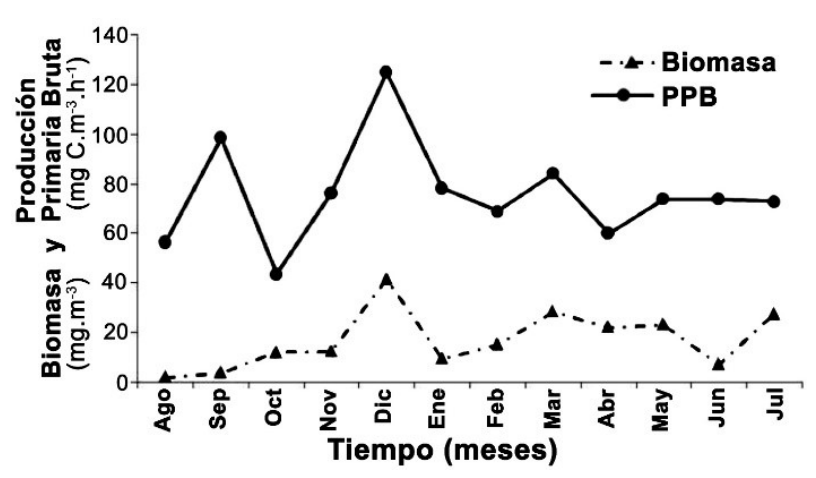

Figura 5. Embalse Riogrande II, Don Matías, Colombia. Variación temporal de la biomasa fitoplanctónica y la producción primaria bruta.

Figure 5. Riogrande II Reservoir, Don Matias, Colombia. Temporal variation of phytoplankton biomass and gross primary production.

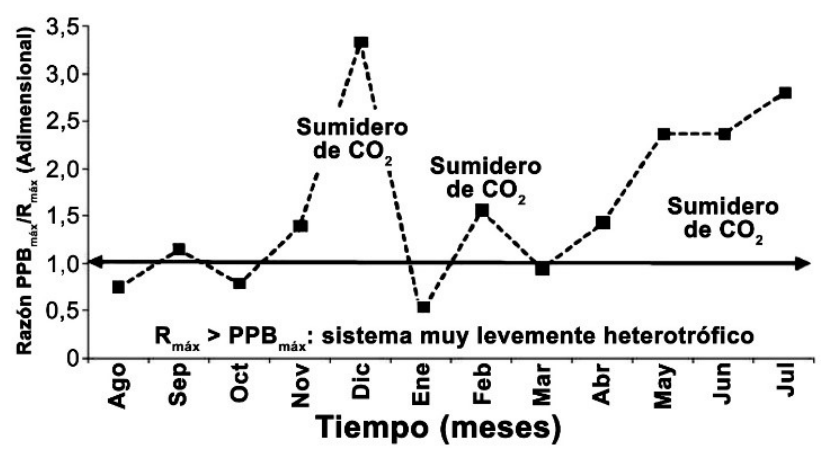

Figura 6. Embalse Riogrande II, Don Matías, Colombia. Variación temporal de la razón $\mathrm{P} / \mathrm{R}$. La flecha constituye el estado estable en el que $\mathrm{PPB}_{\text {máx }}=\mathrm{R}_{\text {máx }}=1 . \mathrm{P}=$ producción; $\mathrm{PPB}=$ producción primaria bruta; $\mathrm{R}=$ respiración.

Figure 6. Riogrande II Reservoir, Don Matias, Colombia. Temporal variation of $P / R$ ratio. The arrow represents the steady state in which $G P P_{\max }=R_{\max }=1 . P=$ production; $P P B=$ gross primary production $;=$ respiration.

Con un valor medio de $8,6 \pm 11,7 \mathrm{mgC}$ (mgCl.a.h) $^{-1}$ y un CV de $136,2 \%$, el coeficiente metabólico (Figura 8) mostró un comportamiento 
temporal bastante similar al de la razón $\mathrm{P} / \mathrm{B}$ (Figura 7): disminución desde el primer mes de colecta hasta el tercero, cuando el sistema fue altamente heterotrófico y se constituyó en una fuente de $\mathrm{CO}_{2}$, para luego disminuir hacia el tercer muestreo y continuar oscilando muy cerca al valor unitario; en este intervalo, el sistema fue levemente heterotrófico y tendió más a la eutrofia.

El valor medio de la razón PPN/PPB fue de 0,2 y su dispersión relativa fue la más alta $(\mathrm{CV}=345,1 \%)$ (Tabla 2). La razón analizada se localiza siempre por debajo del estado estable (Figura 9) lo cual se debe al incremento de $\mathrm{R}$ y a la disminución de PPN (Figura 10).

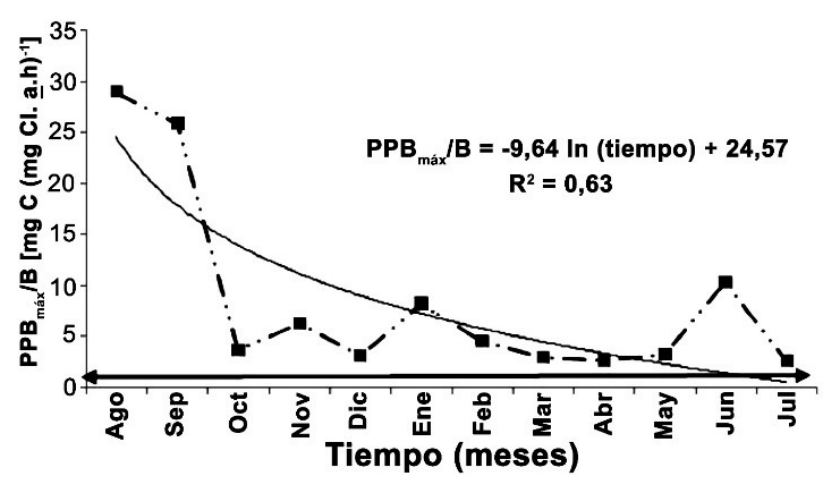

Figura 7. Embalse Riogrande II, Don Matías, Colombia. Variación temporal de la razón P/B. La flecha inferior representa el estado estable, momento en el que PPBmáx $=\mathrm{B}$. $\mathrm{B}=$ biomasa; $\mathrm{P}=$ producción; $\mathrm{PPB}=$ producción primaria bruta; $\mathrm{R}=$ respiración.

Figure 7. Riogrande II Reservoir II, Don Matias, Colombia. Temporal variation of $P / B$ ratio. Lower arrow represents steady state, at which time PPBmáx $=B . B=$ biomass; $P=$ production; $P P B=$ gross primary production; $R=$ respiration .

\section{DISCUSIÓN}

\section{Cinética de los coeficientes, estado estable y aspectos metabólicos}

Para Odum (1972), Biomasa es equivalente a "multitud algal presente". Sin embargo, no debe confundirse "multitud presente" con PP, pues un aumento de B involucra un mayor costo respiratorio para mantener "ordenada" la estructura alcanzada; implica entonces un mayor "bombeo de desorden" y con ello un incremento de R. El hecho de que $\mathrm{R}_{\text {máx }}$ haya sido mayor que $\mathrm{B}$ en $10 \mathrm{de}$ los 12 muestreos (Figura 3), permite suponer que hubo tal eliminación en un esfuerzo por conservar el orden del ensamblaje fitoplanctónico, pero no dice nada acerca de su intensidad, la cual puede deducirse del análisis de la razón P/R. Esta razón, fue mayor que uno en 8 de los 12 muestreos e incrementada notablemente en diciembre y a partir de marzo (Figura 6) cuando la eliminación de desorden o neguentropía (representada por R) se redujo, y se localizó por debajo de $\mathrm{PPB}_{\text {máx }}$ (Figura 5).

La apreciación de Odum (1972) referente a que las comunidades de B considerable requieren una neguentropía considerable para su subsistencia en un estado de orden, permite concluir en primera instancia que la B hallada en esta investigación (media $=17,2$ mgCl.a.m ${ }^{-3}$ ) es alta. Consecuentemente, la razón entre los valores medios de PPB $\left(14,7 \pm 43,2 \mathrm{mgC} \cdot \mathrm{m}^{-3} \cdot \mathrm{h}^{-1}\right)$ y PPN $(75,8 \pm 20,8$ mgC. $\left.\mathrm{m}^{-3} \cdot \mathrm{h}^{-1}\right)$ muestra que PPB fue 5,2 veces PPN, lo que se debe a la mayor R. Estas consideraciones implican en principio que: 1) la estructura de biomasa presente se mantiene dada la neguentropía relativamente alta, según se ha mencionado ya varias veces; y 2) metabólicamente (y dado que $\mathrm{PPB}_{\text {máx }}$ fue tan sólo 2,8 veces mayor que $\mathrm{R}_{\text {máx }}$ ) el sistema es más autotrófico que heterotrófico, comportándose como un sumidero de carbono

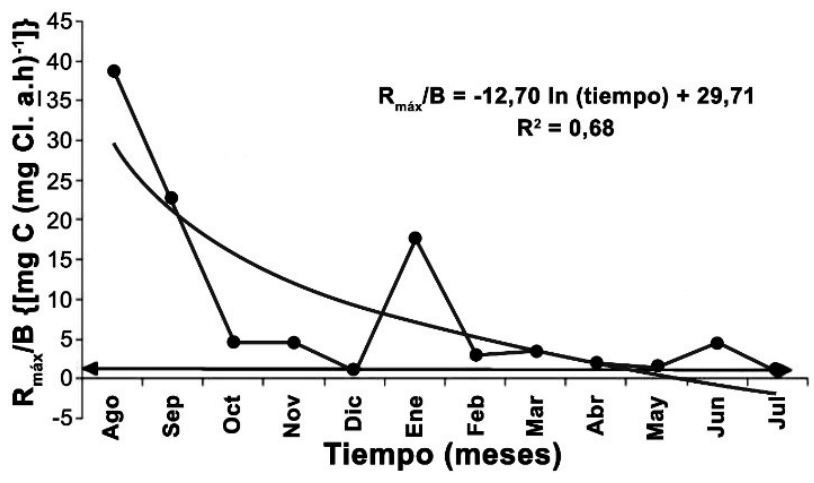

Figura 8. Embalse Riogrande II, Don Matías, Colombia. Variación temporal del coeficiente metabólico. La flecha inferior representa el estado estable cuando Rmáx = B. En el intervalo agosto-septiembre el sistema fue altamente heterotrófico y constituyó una fuente de $\mathrm{CO}_{2}$. De allí en adelante hasta el final del muestreo, su comportamento fue levemente heterotrófico y tendiente a la eutrofía. $\mathrm{B}$ = biomasa; $\mathrm{R}$ = respiración.

Figure 8. Riogrande II Reservoir, Don Matias, Colombia. Temporal variation of metabolic rate. Lower arrow represents the steady state when Rmax $=B$. In the AugustSeptember interval, the system was highly heterotrophic and constituted a source of $\mathrm{CO}_{2}$. From then until the end of sampling period its behavior was slightly heterotrophic and tending to eutrophy. $B=$ biomass; $R=$ respiration. 


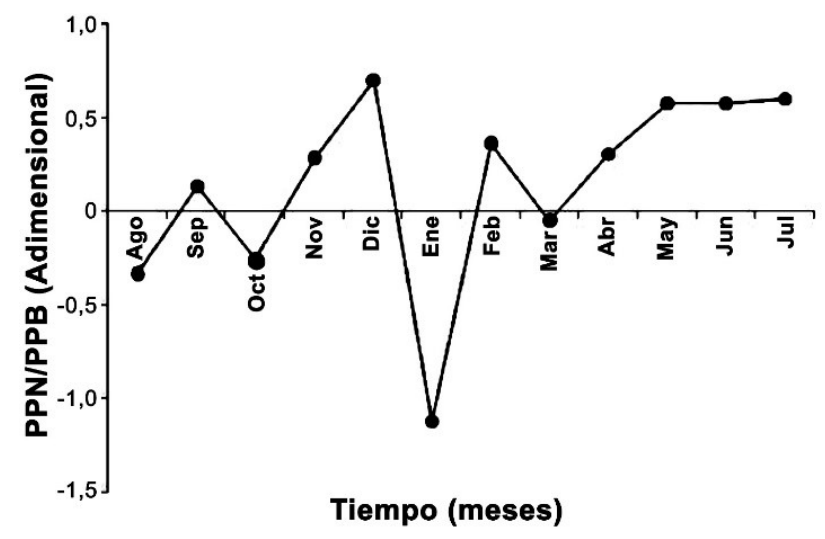

Figura 9. Embalse Riogrande II, Don Matías, Colombia. Variación temporal de la razón PPN/PPB. En esta figura, el valor 1 representa el estado estable; es decir, aquel en el cual $\mathrm{PPN}=\mathrm{PPB}, \mathrm{y} \mathrm{R}=0 \mathrm{PPB}=$ producción primaria bruta; $\mathrm{PPN}=$ producción primaria neta; $\mathrm{R}=$ respiración.

Figure 9. Riogrande II Reservoir, Don Matías, Colombia. Temporal variation of NPP/BPP ratio. In this figure, the value of 1 represents the stable condition, that means that $N P P=G P P$, and $R=0 . P P B=$ gross primary production; $P P N=$ net primary production; $R=$ respiration.

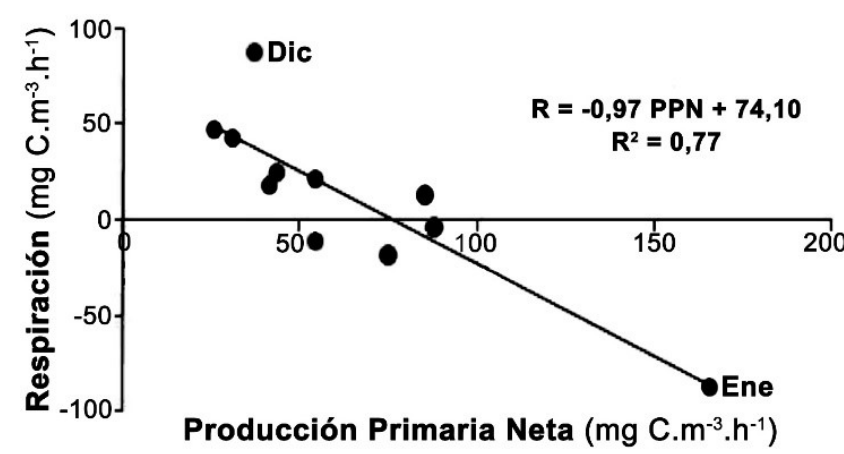

Figura 10. Embalse Riogrande II, Don Matías, Colombia. Respuesta de la respiración en función de los cambios en la producción primaria neta.

Figure 10. Riogrande II Reservoir, Don Matías, Colombia. Response of respiration as a function of changes in net primary production.

en varios intervalos de tiempo. Esta última proporción $\left(2,8 \mathrm{PPB}_{\text {máx }}=\mathrm{R}_{\text {máx }}\right)$ muestra que la intensidad del bombeo neguentrópico para conservar la estructura del ensamblaje no fue tan pronunciada, pues de ser así sucedería lo contrario $(\mathrm{PPB}<\mathrm{R})$. Además, los valores negativos de PPN, que sugieren valores altos de R (Figuras 3 y 5), se presentaron sólo en el mes de enero (Figura 10).

Duarte \& Agustí (1998), plantean que los sistemas productivos (eutróficos) - como el em- balse Riogrande II-son sistemas autotróficos, mientras que los poco productivos son más heterotróficos requiriendo insumos alóctonos de carbono para poder soportar la alta R (Cole 1999). Inicialmente se supuso que a la represa del embalse no alcanzarían a llegar los aportes alóctonos ni los autóctonos y, por ello, la producción de B sería baja; esta presunción no resultó cierta para este trabajo.

Para Harris (1978) el coeficiente metabólico fluctúa entre 0,6 y 3,0 mgC (mgcl.a.h) ${ }^{-1}$; en nuestro caso varió desde 0,9 a 38,6 con un valor medio de $8,6 \pm 11,7$ y una variabilidad bastante alta (Tabla 2). Salvo los meses de agosto y septiembre de 2002 y enero de 2003 el coeficiente osciló levemente alrededor de la unidad, confirmando la relativa estabilidad del ensamblaje fitoplanctónico en el sitio de muestreo, con algunos periodos catabólicos, pero primando los de carácter anabólico, lo que efectivamente convierte el sistema en un sumidero de carbono capaz de mantenerse en condición estable y, por tanto, resistente a los aportes entrópicos o anérgicos desordenadores provenientes de sus alrededores. Este escenario concuerda plenamente con lo formulado por Odum (1969) en lo que se refiere a que, en los ecosistemas maduros, la entropía es baja y la información contenida en la B alta. Información se refiere a los efectos que confieren orden a un sistema (Angelini \& Gomes 2008).

A medida que un ecosistema madura y se desarrolla, cabe esperar que la asimilación fotosintética y el coeficiente metabólico tiendan a decrecer (Odum 1969). Reynolds (2006) considera además que los ecosistemas maduros evolucionan tanto cuanto se esfuerzan en optimizar la ascendencia para llegar al estado de clímax, siendo la ascendencia, al igual que la información una medida de ordenación. De acuerdo con este postulado, el sistema embalse Riogrande II se halla en el estado estable (Figuras 7 y 8) y es un sistema que tiende a acumular información bajo la forma de B no respirada. No obstante, en nuestro caso es necesario dejar claro que, contrario a lo afirmado por Odum (1969), la razón P/R se incrementó hacia el final del muestreo (Figura 6) alejándose del valor estable $(\mathrm{P} / \mathrm{R}=1)$ postulado por él como característico de ecosistemas maduros. Lo anterior corrobora lo ya afirmado varias veces en este documento en cuanto a que el 
bombeo neguentrópico realizado por $\mathrm{R}$ no es tan alto como para respirar toda la B presente quedando energía útil o disponible (exergía) no usada (= emergía) que puede ser aprovechada dentro del sistema en el sitio de muestreo y que aún podría ser respirada. $\mathrm{Si}$ aceptamos que la energía utilizable, disponible o exergía está asociada al desequilibrio entre un sistema y su entorno, podemos pensar que el sitio estudiado en el embalse Riogrande II posee una alta cantidad de ella.

Según Margalef (1974) el coeficiente de asimilación fotosintética vale para el plancton 3,7 tendiendo a 5,0 con valores superiores ocasionales. La eficiencia fotosintética media de Riogrande II fue de 8,5 \pm 9,2 mgC. (mgCl.a.h) ${ }^{-1}$, lo cual equivale a decir que por cada gramo de clorofila a, se asimilaron $0,85 \mathrm{~g}$ de carbono por hora, cifra 5,7 veces superior al valor medio para los 14 embalses estudiados (0,15 gC por cada gCl.a; Tabla 3). Margalef (1993) establece que $1 \mathrm{~g}$ de clorofila a absorbe un máximo de 25 gC.h ${ }^{-1}$, con un máximo observado de 11 gC.h ${ }^{-1}$ y valores comunes entre $3 \mathrm{y}$ 4 gC.h ${ }^{-1}$ (Margalef 1993). Las cifras halladas en Riogrande II fueron bastante inferiores a las citadas, quizás debido a la turbidez del embalse y a la poca penetración de luz en el mismo inducido por la alta B presente, según se planteó hipotéticamente en la introducción.

La asimilación fotosintética de Riogrande II (Tabla 3) fue muy superior a la citada para cualquiera de los 14 embalses brasileros presentados, a pesar de la limitación en el clima óptico del sitio muestreado, lo cual se discutirá más ampliamente en parágrafos posteriores. Los valores de $\mathrm{P} / \mathrm{B}, 8,5$ veces superiores a la unidad, corroboran la condición autotrófica del embalse. Margalef (1993) considera que la concentración de clorofila a por unidad de área fotosintética no excede los 400 mg.m ${ }^{-2}$, quedando siempre por debajo de este valor; en nuestro caso, el valor medio estimado es de 392,5, bastante cercano al referenciado por Margalef (1993).

$\mathrm{Si}$ asumimos que $\mathrm{B}$ acumulada significa incremento de $R$, entonces $P / R$ ha de ser menor que uno en esta investigación. No obstante, la razón $\mathrm{P} / \mathrm{R}$, con un valor medio de 1,61 muestra claramente un desbalance entre el material producido (orden) y el eliminado o consumido por $\mathrm{R}$ (neguentropía), lo que implica que por mucho que respire el sistema, no alcanza a consumir lo que le llega. Esto corrobora que el sistema es más del tipo autotrófico y no consume la $\mathrm{P}$ propia, resultante del proceso de disipación del exceso de entropía entrante proveniente de los alrededores de la cuenca funcionando como un sumidero de C; esto muestra que dichos alrededores son una fuente grande de entropía que es 'negada', bombeada o disipada en el interior del mismo por el ensamblaje algal (y bacteriano) presente; pero este bombeo respiratorio no alcanza a eliminar el exceso de desorden que penetra el embalse, sobrando carbono para el resto de los componentes de la trama trófica. Odum (1972) menciona que, en la mayoría de los sistemas naturales, la intensidad de $\mathrm{P}$ pasa de un equilibrio de estado estable temporal a otro, a causa de los cambios impuestos en el sistema; aunque el embalse como tal no es un sistema natural, su devenir se ajusta a esta premisa. Dado que este sistema se localiza en la zona ecuatorial, con una alta entrada de radiación y temperaturas altas, se espera una tasa respiratoria alta y con ella un bombeo de desorden también alto. De hecho, es así, pero como ya fue mencionado, de los alrededores entra un exceso de entropía-que representa subsidios de energía reforzadores de la producción-,lo que reduce la pérdida o bombeo de calor respiratorio eliminando el desorden para mantener así un ensamblaje estable y disponible bajo la forma de $\mathrm{B}$, y en última instancia de carbono, representado por la PPN.

La PPN es la producción que queda verdaderamente a disposición de los siguientes niveles tróficos (Odum 1972, Margalef 1974, 1983). Si consideramos los valores medios de PPB $(=75,8$ mgC. $\left.\mathrm{m}^{-3} \cdot \mathrm{h}^{-1}\right)$ y los de R $\left(=61,1 \mathrm{mgC} \cdot \mathrm{m}^{-3} \cdot \mathrm{h}^{-1}\right), \mathrm{y}$ asumimos que PPB $=\mathrm{PPN}+\mathrm{R}=75,8 \mathrm{mgC} \cdot \mathrm{m}^{-3} \cdot \mathrm{h}^{-1}$, podemos calcular que $\mathrm{R}$ es el $80,6 \%$ de $\mathrm{PPB}$, restando $14,7 \%$ de PPN como P disponible para el resto de los componentes de la trama trófica. Esto reafirma que el sistema embalse Riogrande II es un sumidero de carbono y que dicho sumidero está representado en el ensamblaje fitoplanctónico mediante P. Por tanto, en la escala anual, el sistema se encuentra alejado del estado estable una buena parte del tiempo muestreado.

Según Schindler (1978), en los lagos altamente productivos el valor de $\mathrm{R}$ es generalmente una alta proporción de PPB debido a que la razón $\mathrm{D}_{\text {eu }} / \mathrm{D}_{\mathrm{mix}}$ es típicamente baja en estos lagos, llegando en 
Tabla 3. Valores del coeficiente de asimilación citados por autores varios para algunos lagos y embalse ecuatoriales, tropicales y subtropicales.

Table 3. Assimilation coefficient values cited by several authors for some equatorial, tropical and subtropical lakes and reservoir.

\begin{tabular}{|c|c|c|}
\hline Sistema & $\begin{array}{l}\text { Asimilación fotosintética } \\
\left.\text { [mgC. }(\text { mgCl.a.h })^{-1}\right]\end{array}$ & Autor \\
\hline \multicolumn{3}{|l|}{ LAGOS } \\
\hline Lago Dom Helvecio (Brasil) & 0,99 & Tundisi \& Tundisi (2008) \\
\hline Lago Jacaré (Brasil) & 0,23 & Tundisi \& Tundisi (2008) \\
\hline Lagoa Carioca (Brasil) & 0,35 & Tundisi \& Tundisi (2008) \\
\hline Lago George (Uganda) & 8,30 & Westlake et al. (1980) \\
\hline Lago do Castanho (Brasil) & 15,6 & Westlake et al. (1980) \\
\hline \multicolumn{3}{|l|}{ EMBALSES } \\
\hline Barra Bonita & 2,56 & Tundisi (1983) \\
\hline Ibitinga & 2,16 & Tundisi (1983) \\
\hline Promissão & 0,83 & Tundisi (1983) \\
\hline Capivara & 3,40 & Tundisi (1983) \\
\hline Salto Grande & 2,07 & Tundisi (1983) \\
\hline Jurumirim & 1,02 & Tundisi (1983) \\
\hline Volta Grande & 1,22 & Tundisi (1983) \\
\hline Ponte Nova & 0,49 & Tundisi (1983) \\
\hline Pedreira & 2,45 & Tundisi (1983) \\
\hline Riacho Grande & 1,20 & Tundisi (1983) \\
\hline Rio das Pedras & 1,64 & Tundisi (1983) \\
\hline Guarapiranga & 1,09 & Tundisi (1983) \\
\hline Americana & 0,91 & Tundisi (1983) \\
\hline Paraibuna & 0,38 & Tundisi (1983) \\
\hline Esta investigación & 8,70 & \\
\hline Media para 14 embalses & 1,53 & \\
\hline D (para embalses) & 4,55 unidades & \\
\hline
\end{tabular}

muchos de ellos a ser R hasta el 50\% o más de PPB. Pero aquí, la razón mencionada se situó muy cerca de la unidad (media $=1,13$ ) por lo que los altos valores de $\mathrm{R}$ pueden deberse más a la temperatura relativamente alta del agua en la zona de represa (media $=20,5 \pm 0,9^{\circ} \mathrm{C}$ ) que incrementa la $\mathrm{R}$ del ensamblaje, a pesar que las algas no hayan pasado mucho tiempo en la oscuridad. Por ello, confirmamos que la premisa referente al no aporte autóctono ni alóctono a la represa es falsa. Quizás no sobra recordar que según Powell (1989), la $\mathrm{D}_{\text {mix }}$ representa "una breve ventana en el tiempo" que afecta el reclutamiento de nuevos nutrientes que son cargados hasta la zona iluminada donde si hay un suplemento lumínico adecuado podrán ser usados para la producción fotosintética para luego ser respirada. Dado que en Riogrande II la profundidad de mezcla es casi igual a la profundidad fótica, el fitoplancton debe residir porciones de tiempo similares en los estratos iluminados, por lo que su exposición a la luz no es pronunciada y puede no incrementar la fotorespiración; quizás por ello los valores de la razón $\mathrm{P} / \mathrm{R}$ fueron superiores a uno la mayor parte del tiempo (Figura 6).

La razón $\mathrm{P} / \mathrm{R}$ determina si el fitoplancton puede o no sobrevivir en una capa mezclada. A partir de ella, Talling $(1957,1965,1971)$ propone los denominados modelos de "Razón Crítica" $\left(\mathrm{q}_{\mathrm{c}}\right)$ entendidos como aquellos que permiten calcular la profundidad de mezcla que asegura que las pérdidas respiratorias (neguentropía) están balanceadas por las ganancias fotosintéticas. En aquellos periodos en que $\mathrm{q}_{\mathrm{c}}$ es menor que la unidad, el bombeo neguentrópico, eliminador de desorden y representado por $\mathrm{R}$, es mayor que el proceso originador del mismo (la P), la sobrevivencia del ensamblaje algal está limitada especialmente por luz; en los periodos en que ocurre lo contrario, la supervivencia de estos organismos es mayor (Harris 1978). Consecuentemente, en el embalse Riogrande II, este 
ensamblaje tendría problemas en los periodos agosto-septiembre y enero-abril (Figura 11), cuando el proceso catabólico prima sobre el anabólico $\left(\mathrm{q}_{\mathrm{c}}<1\right)$ y la pluviosidad va en aumento (Figura 2). Es menester resaltar que en esta investigación la razón $\mathrm{D}_{\mathrm{eu}} / \mathrm{D}_{\mathrm{af}}$ (razón profundidad fótica-profundidad afótica) fue siempre inferior a uno (media $=0,2 \pm 0,1 ; V_{\text {mín. }}=0,1 ; V_{\text {máx }}=0,3$; $\mathrm{D}_{\text {eu }} / \mathrm{D}_{\mathrm{af}}=-0,01$ tiempo $\left.+0,25 ; \mathrm{R}^{2}=0,7 ; \mathrm{p}=0,005\right)$, por lo que la mayor parte del tiempo, la zona sin luz fue mayor que la iluminada; en esta zona es donde ocurre el proceso catabólico del metabolismo.

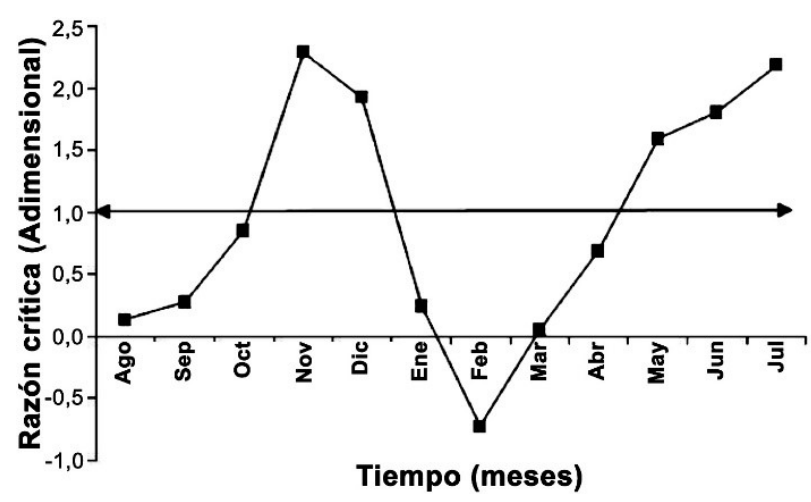

Figura 11. Embalse Riogrande II, Don Matías, Colombia. Variación de la razón crítica en función de los cambios en una escala temporal anual. La flecha señala el estado estable en que PPB por día en términos de área=Respiración por día calculada en los mismos términos. $\mathrm{PPB}=$ producción primaria bruta.

Figure 11. Riogrande II Reservoir, Don Matías, Colombia.Variation of critical rate in terms of changes in an annual time scale. The arrow points to the steady condition in which $P P B$ per day in terms of area = Respiration per day calculated in the same terms. $P P B=$ gross primary production.

La B fitoplanctónica expresada en mgCl.a por unidad de área fotosintetizante alcanza un valor máximo que oscila entre 200 y 500 mgCl.a.m ${ }^{-2}$ (Steemann Nielsen 1952, Ahlgren 1970, Talling 1970, Margalef 1974); por encima de esta cantidad de clorofila no habrá ningún incremento en la fotosíntesis por unidad de área debido a la reducción de la zona fotosintética por el autosombreo algal ("algal self-shading”) (Beadle 1974). Por ello, Ahlgren (1970) y Talling (1970) establecen que en el intervalo señalado de clorofila a por $\mathrm{m}^{2}$, la limitación es más por luz que por nutrientes. Valores de $300 \mathrm{mgCl}^{\mathrm{a} . \mathrm{m}^{-2}}$ son referenciados por Margalef (1974) como suficientes para absorber prácticamente toda la radiación eficaz, permitiendo fijar máximo entre 1000 y $1500 \mathrm{mg} \cdot \mathrm{m}^{-2} \cdot \mathrm{h}^{-1}$, para un coeficiente de asimilación fotosintética localizado entre 3,0 y 5,0 mgC(mgCl.a.h) $)^{-1}$ cuando computado a partir de términos por unidad de área. Aunque el valor medio de la B fitoplanctónica por unidad de área fotosintética hallado en este estudio (392,5 $\pm 269,7$ mgCl.a.m ${ }^{-2}$ ) se encuentra en el intervalo sugerido (200 y 500 mgCl.a.m ${ }^{-2}$ ), el de PPB máx $(104,7 \pm 69,3$ mgC. $\mathrm{m}^{-2} \cdot \mathrm{h}^{-1}$ ), fue aproximadamente 12 veces inferior al aludido; por ello, en principio concluimos que en la represa del embalse el ensamblaje fitoplanctónico está limitado por luz y que esta restricción parece ser la causa de los valores muy inferiores de PPB cuando comparados con los presentados por Margalef (1974), lo que concordaría plenamente con las limitantes que la condición trófica del embalse le impondría a la penetración de luz. Al comparar los resultados del Riogrande II con los de embalses brasileros (Tabla 4), se concluye que el cuerpo de agua bajo consideración es bastante disímil con respecto a la concentración de clorofila, pero muy similar a los demás en lo referente a la PPB.

El coeficiente de autosombreo algal $\left(K_{s}\right)$ correspondió a $0,011 \mathrm{~m}^{2} \cdot \mathrm{mgCl} \cdot \mathrm{a}^{-1}$ (Figura 12), localizado en el intervalo citado por Talling (1971) $(0,010-0,020)$ y por Harris et al. (1980) $(0,017-0,011)$. Este valor muestra que por $\mathrm{m}^{2}$ de zona iluminada hay mucha clorofila $\left(\right.$ media $=392,5 \mathrm{mg} \cdot \mathrm{m}^{-2}$ ). La pregunta es si esta cantidad de clorofila impide realmente la entrada de luz, lo cual depende del tamaño de las algas y de la forma como estén empaquetadas las unidades de clorofila. Según Harris et al. (1980), en los lagos con una columna de agua ópticamente profunda $\left(\mathrm{D}_{\mathrm{eu}}>\mathrm{D}_{\text {mix }}\right)$ se desarrollan durante periodos estables de $\mathrm{D}_{\text {eu }} / \mathrm{D}_{\text {mix }}$ $(\approx 1)$ poblaciones de células grandes que no impiden la entrada de luz; y cuando ocurren perturbaciones físicas que alteran la razón, hay un cambio hacia pequeñas células, más adaptadas a soportar variaciones en los niveles de luz en la columna de agua. Como se ha mencionado ya, en el cuerpo de agua estudiado la razón $D_{\text {eu }} / D_{\text {mix }}$ presentó valores cercanos a uno durante todo el tiempo de muestreo (media $=1,13 \pm 0,32$ ) lo que corresponde con lo planteado por el autor citado. Posiblemente por ello, la clorofila en el embalse se 
Tabla 4. Valores de la concentración de clorofila a en términos de área, y de producción primaria bruta diaria para lagos y embalses ecuatoriales, tropicales y subtropicales según varios autores.

Table 4. Values of chlorophyll a concentration in terms of area, and daily gross primary production equatorial, tropical and subtropical lakes and reservoirs according to several authors.

\begin{tabular}{|c|c|c|c|}
\hline Lago & mgCl.a.m-2 & PPB (gC.m-2.d-1) & Autor \\
\hline \multicolumn{4}{|l|}{ LAGOS } \\
\hline Victoria (Uganda) & $35,0-100,0$ & $1080,0-4200,0$ & Talling (1976) \\
\hline Kivu (Zaire) & - & 1440,0 & Degens et al. (1971) \\
\hline Mulehe (Uganda) & - & 960 & Talling (1965) \\
\hline Bunyoni (Uganda) & & 1800,0 & Talling (1965) \\
\hline George (Uganda) & $70,0-280,0$ & 540,0 & Ganf (1975) \\
\hline Chad (El Cairo) & - & $700,0-2700,0$ & Lemoalle (1969) \\
\hline Aranguadi (Ethiopia) & $221,0-325,0$ & $13000,0-22000,0$ & Baxter et al. 1965 \\
\hline Nakuru (Kenia) & - & $2300,0-3200,0$ & Melack \& Kilham (1974) \\
\hline Ciénaga Grande (Colombia) & $9,0-290,0$ & $1,4-16,3$ & Hernández \& Gocke (1990) \\
\hline \multicolumn{4}{|l|}{ EMBALSES } \\
\hline Barra Bonita & 15,9 & 398,3 & Tundisi (1983) \\
\hline Ibitiunga & 29,8 & 483,9 & Tundisi (1983) \\
\hline Promissão & 68,7 & 584,1 & Tundisi (1983) \\
\hline Capivara & 12,7 & 188,7 & Tundisi (1983) \\
\hline Salto Grande & 5,7 & 102,8 & Tundisi (1983) \\
\hline Jurumirim & 9,7 & 103,0 & Tundisi (1983) \\
\hline Volta Grande & 31,7 & 340,2 & Tundisi (1983) \\
\hline Ponte Nova & 76,1 & 331,4 & Tundisi (1983) \\
\hline Pedreira & 94,7 & 1913,9 & Tundisi (1983) \\
\hline Riacho Grande & 208,1 & 1466,8 & Tundisi (1983) \\
\hline Rio das Pedras & 132,6 & 1371,0 & Tundisi (1983) \\
\hline Guarapiranga & 7,8 & 50,1 & Tundisi (1983) \\
\hline Americana & 113,8 & 786,0 & Tundisi (1983) \\
\hline Paraibuna & 16,8 & 43,8 & Tundisi (1983) \\
\hline Esta investigación & 392,5 & 1256,5 & \\
\hline D para embalses & 5,7 unidades & - 0,7 unidades & \\
\hline
\end{tabular}

$\overline{\mathrm{D}}=(\mathrm{xi}-\overline{\mathrm{X}}) / \overline{\mathrm{X}}($ Hakanson 1981): desviación del valor medio obtenido en este estudio en relación al valor de la media aritmética. / $D=(x i-\bar{X}) / \bar{X}$ (Hakanson 1981): deviation of the mean value obtained in this study in relation to the arithmetic mean value.

reúne en paquetes grandes con células voluminosas en su interior y con bajos valores de $\mathrm{K}_{\mathrm{s}}$ por lo que no consiguen atenuar suficientemente la entrada de luz. Ramírez-Restrepo et al. (2015) probaron que en el embalse Riogrande II, la dispersión es más por el tripton que por el fitoplancton como partícula, lo que concuerda con lo afirmado por Porras-Zapata et al. (1997) en lo referente a su turbidez relativamente alta (media $=$ $188 \pm 13,8 \mathrm{NTU} ; \mathrm{N}=35$ ) que sería, más que la $\mathrm{B}$ fitoplanctónica, el factor que impediría la penetración de luz.

El resultado de la proporción profundidad fótica $\left(D_{\text {eu }}\right)$ y profundidad de mezcla $\left(D_{\text {mix }}\right)$ representa la fracción de la capa mezclada que es iluminada por radiación mayor que $1 \%$ del valor de $\mathrm{I}_{0}$ ' en la superficie. $\mathrm{Si} \mathrm{D}_{\text {mix }}>\mathrm{D}_{\text {eu}}$, entonces el

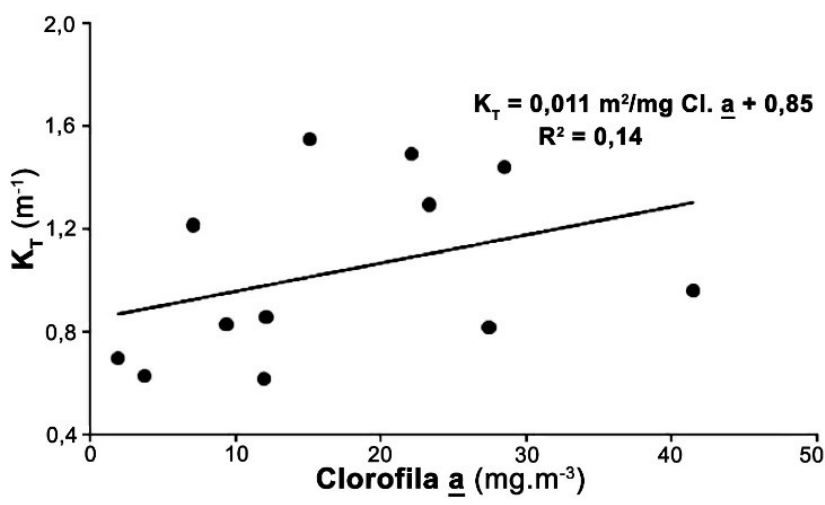

Figura 12. Embalse Riogrande II, Don Matías, Colombia. Relación entre el coeficiente de atenuación total en función de la concentración de clorofila a.

Figure 12. Riogrande II Reservoir, Don Matías, Colombia. Relationship between total attenuation coefficient as a function of chlorophyll a concentration. 
fitoplancton pasará más tiempo en la oscuridad; por tanto, la neguentropía ejercida por $\mathrm{R}$ se incrementará. Si aceptamos que $\mathrm{P}$ es la cantidad de $B$ ensamblada y acumulada mediante fotosíntesis, y a ella le sumamos la pérdida de la R; y si además asumimos que $\mathrm{P}$ es ganancia de orden o flujo de exergía en el sistema; y que B acumulada significa incremento potencial de R; y que cuando se alcanza el estado estable el componente biótico "cosecha" el flujo entero de energía, la disipación entrópica interna del organismo $\left(\mathrm{dS}_{\mathrm{e}}\right)$ tiende a cero $\left(\mathrm{dS}_{\mathrm{e}} \rightarrow 0\right)$ y consume esa energía exclusivamente en su propio mantenimiento (la disipación interna $d S_{i}=R \rightarrow 0$ ); y que cuando esto ocurre ningún incremento es posible (Reynolds 2006), entonces la suma de $\mathrm{B}$ y la de $\mathrm{R}$ acrecerán a medida que la razón $\mathrm{D}_{\text {eu }} / \mathrm{D}_{\text {mix }}$ decrezca debido al incremento de la $\mathrm{D}_{\text {mix }}$, el incremento de $\mathrm{R}$ y el decrecimiento de B.

En nuestro caso, la regresión $\mathrm{D}_{\text {eu }} / \mathrm{D}_{\text {mix }}-\Sigma(\mathrm{B}$ y R $)$ se ajustó a una función lineal $\left(\mathrm{D}_{\mathrm{eu}}=0,55 \mathrm{D}_{\text {mix }}+2,32\right.$, $\mathrm{R}^{2}=0,65, \mathrm{p}=0,001$ ), lo que significa que a medida que se incrementa $D_{\text {mix, también lo hace la }}$ penetración de luz, sin que exista propiamente una zona oscura de mayor $\mathrm{R}$ para las algas. Por ello, no se presentó una relación significativa entre la suma $(B+R)$ y la razón $D_{\text {eu }} / D_{\text {mix }}$ pues a medida que se incrementaba la razón (esto es, decae la $\mathrm{D}_{\text {mix }}$, la línea de mejor ajuste es casi asintótica al eje de las abscisas. Esto significa que dicha suma no se afecta con ese cambio debido a que el ensamblaje se halla cercano al estado estable, $\mathrm{R} \rightarrow$ 0 y sólo queda la $\mathrm{B}$ que no puede respirarse y que queda incluida como PPN. Recuérdese que: dS (disipación entrópica total $)=\mathrm{dS}_{\mathrm{i}}(=\mathrm{R})+\mathrm{dS}_{\mathrm{e}}\left(=\sum \mathrm{B}\right)$, si $\mathrm{dS}_{\mathrm{e}} \rightarrow 0$, entonces $\mathrm{R}=\mathrm{dS} \mathrm{S}_{\mathrm{i}} \rightarrow \mathrm{dS}$, y R asume el papel de la disipación entrópica total o neguentropía .

Aunque en principio todo apunta a que en la represa del embalse el ensamblaje fitoplanctónico está limitado por luz y que esta restricción parece ser la causa de los valores muy inferiores de PPB, no estamos totalmente de acuerdo con lo previsto ya que, aunque hay obvias restricciones lumínicas, el hipolimnio, a pesar de su relativo aislamiento por la estatificación térmica presente, podría por el movimiento interno de las corrientes de agua provenientes de los ríos Chico y Grande, llevar nutrientes a la zona fótica con lo que la $\mathrm{P}$ se incrementaría, especialmente durante los perio- dos lluviosos. Igualmente, las oscilaciones en la profundidad de la $\mathrm{D}_{\text {mix }}$ representarían aportes de nuevos nutrientes a la zona iluminada, los cuales con un suplemento lumínico adecuado podrán ser usados para la absorción de Carbono.

\section{Clasificación trófica}

Considerando la concentración de B fitoplanctónica, la represa del cuerpo de agua estudiado es eutrófica. Esta afirmación está respaldada por el ámbito de variación citado por Esteves (1998): 0,3 a $3 \mathrm{mgCl} . \mathrm{a}^{-3}$ (oligotrófico), 2 a 15 (mesotrófico), 15 a 500 (eutrófico). El valor medio encontrado $\left(17,1 \pm 11,7\right.$ mgCl.a.m $\left.{ }^{-3}\right)$ lo sitúa en este esquema como eutrófico.

En cuanto al coeficiente de asimilación, Tundisi \& Tundisi (2008) establecen valores mayores de 5 mgC.(mgCl.a.h) ${ }^{-1}$ como indicadores de sistemas eutróficos; la represa del embalse ostentó valores medios de 8,5 $\pm 9,2$, que confirman su condición eutrófica. Tundisi \& Tundisi (2008) refieren valores superiores a $200 \mathrm{mgC} \cdot \mathrm{m}^{-3} \cdot \mathrm{d}^{-1}$ para lagos eutróficos; el sistema estudiado muestra valores medios superiores al citado (1256,5 \pm $831,4)$

Para Margalef (1993), la P anual de los ecosistemas acuáticos continentales eutróficos se sitúa entre los 80 y los 500 gC.m ${ }^{-2} \cdot \mathrm{a}^{-1}$, con una media de aproximadamente 300 y una varianza por demás grande. Comparando el valor de $\Sigma \sum \mathrm{PPB}$ citado por Margalef (1993) con el obtenido (437,3 gC. $\left.\mathrm{m}^{-2} \cdot \mathrm{a}^{-1}\right)$, la represa continúa siendo eutrófica. Para Boynton et al. (1982), los sistemas estuarinos-altamente productivos por demás-presentan una $\mathrm{P}$ media de $256 \mathrm{gC} . \mathrm{m}^{-2} \cdot \mathrm{a}^{-1}$, la cual es casi la mitad de la obtenida en esta investigación. La Ciénaga Grande de Santa Marta (Colombia), un sistema estuarino caribeño, muestra valores bastante altos de PPB: $1690 \mathrm{gCm}^{-2} \cdot \mathrm{a}^{-1}$ (Hernández \& Gocke 1990); pero la laguna costera Barra de Navidad, en el Océano Pacífico mexicano, presenta valores medios de 430 (Sandoval Rojo et al. 1988). Cifras muy similares muestra la laguna del Río Cuarto, en Costa Rica $\left(\mathrm{PPB}\right.$ anual $\left.=470 \mathrm{gC} \cdot \mathrm{m}^{-2} \cdot \mathrm{a}^{-1}\right)$ catalogada por Gocke et al. (1990) como un sistema eutrófico; estos dos últimos estimativos son muy similares a los obtenidos en Riogrande II. Además, entre varios lagos tropicales el embalse Riogrande II destaca como uno de los más productivos y muy poco desviado del valor medio (Tabla 5). 
Tabla 5. Valores de $\Sigma \Sigma \mathrm{PPB}$ referidos por Westlake et al. (1980), Talling (1965), Ganf (1975) y Esteves (1998) para algunos lagos ecuatoriales y tropicales.

Table 5. PPB values reported by Westlake et al. (1980), Talling (1965), Ganf (1975) and Esteves (1998) for some equatorial and tropical lakes.

\begin{tabular}{|c|c|}
\hline Lago & gC.m $m^{-2} \cdot a^{-1}$ \\
\hline Ramgardh (India) & 186,6 \\
\hline Sidddanath (India) & $13,3-500,0$ \\
\hline Do Castanho (Brasil) & $350,0-1500,0$ \\
\hline Chilwa (Malawi) & $243,3-433,3$ \\
\hline Victoria (África) & 950,0 \\
\hline George (Uganda) & 1980,0 \\
\hline Dom Helvecio (Brasil) & 68,0 \\
\hline Represa do Lobo (Brasil) & 56,0 \\
\hline Cristalino (Brasil) & $53,0-1500,0$ \\
\hline Lanao (Filipinas) & 620,0 \\
\hline Tupé (Brasil) & 100,0 \\
\hline Kiwu (Zaire) & 540,0 \\
\hline Este estudio & 437,3 \\
\hline $\mathrm{D}$ & - 0,4 unidades \\
\hline \multicolumn{2}{|c|}{$\begin{array}{l}\mathrm{D}=(\mathrm{xi}-\overline{\mathrm{X}}) / \overline{\mathrm{X}} \text { (Hakanson 1981): desviación del valor } \\
\text { medio obtenido en este estudio en relación al valor de la } \\
\text { media aritmética. / } D=(x i-\bar{X}) / \bar{X} \text { (Hakanson 1981): de- } \\
\text { viation of the mean value obtained in this study in } \\
\text { relation to arithmetic mean value. }\end{array}$} \\
\hline
\end{tabular}

Por su parte, Esteves (1998) identifica como lagos euproductivos a aquellos con valores superiores a $500 \quad \mathrm{gC} \cdot \mathrm{m}^{-2} \cdot \mathrm{a}^{-1}$, como mesoproductivos a los que poseen valores entre 200 y 500, y como oligoproductivos a los que presentan cifras inferiores a $200 \mathrm{gC} . \mathrm{m}^{-2} \cdot \mathrm{a}^{-1}$. Los valores medios hallados en la represa del embalse $(437,3)$ lo identifican como muy cercano a un sistema euproductivo. Incluso cuando comparado con los valores citados por Esteves (1998) para lagos templados de distintos estados tróficos, sólo uno de ellos es mayor que la $\Sigma \Sigma$ PPB presentada por el embalse investigado (Tabla 6).

Esteves (1998) cita además para lagos de zona templada intervalos de PP diaria entre 50 y 300 mgC. $\mathrm{m}^{-2} \cdot \mathrm{d}^{-1}$ para sistemas oligotrófico, entre 250 1000 para mesotróficos, mayores de 1000 para eutróficos, y menores de 50-500 para lagos distróficos. Aunque ecuatorial, puede decirse que la represa del embalse Riogrande II, con una PPB media de 1072,8 $\pm 1256,5 \mathrm{mgC} \cdot \mathrm{m}^{-2} \cdot \mathrm{d}^{-1}$, muestra valores muy superiores a los citados por Esteves en 1998 para los lagos templados eutróficos. Igualmente, al comparar la PPB diaria con los datos obtenidos en embalses venezolanos por
González et al. (2003) y en la laguna del Río cuarto vemos que, con excepción de tres de ellos, los valores hallados en el embalse estudiado son relativamente cercanos a los mostrados por algunos de los cuerpos de agua (Tabla 7).

Tabla 6. Valores de producción primaria bruta anual para lagos de latitudes altas.

Table 6. Annual gross primary production values for high latitude lakes.

\begin{tabular}{lc}
\hline Lago & $\mathbf{g C . m}^{-\mathbf{2}} \cdot \mathbf{a}^{-\mathbf{1}}$ \\
\hline Vorderer Finstaler (Austria) (1) & $23,0-31,0$ \\
\hline Heindalsvatm (Noruega (1) & 6,9 \\
\hline Schöh (Alemania) (2) & 105,0 \\
\hline Keller (Alemania) (3) & 250,0 \\
Trumen (Suecia) (3) & 260,0 \\
\hline Sylven (Estados Unidos) (4) & 570,0 \\
\hline Este estudio & 437,3 \\
\hline \multicolumn{1}{c}{ D } & 1,1 unidades \\
\hline
\end{tabular}

(1) Oligotrófico, (2) Mesotrófico, (3) Eutrófico, (4) Hipereutrófico. $\quad \mathrm{D}=(\mathrm{xi}-\overline{\mathrm{X}}) / \overline{\mathrm{X}}$ (Hakanson 1981): desviación del valor medio obtenido en este estudio en relación al valor de la media aritmética. / (1) Oligotrophic, (2) Mesotrophic, (3) Eutrophic, (4) Hipereutrophic. $D=(x i-\overline{\mathrm{X}}) / \overline{\mathrm{X}}$ (Hakanson 1981): deviation of the mean value obtained in this study in relation to arithmetic mean value.

Tabla 7. Valores de producción primaria bruta diaria en cinco embalses venezolanos y el embalse Riogrande II.

Table 7. Daily gross primary production values in five Venezuelan reservoirs and in Riogrande II reservoir.

\begin{tabular}{lc}
\hline Embalse & gC.m-2.d-1 $^{-\mathbf{1}}$ \\
\hline Pao Cachinche & $2,50-3,20$ \\
La Mariposa & 1,60 \\
El Lagartijo & 1,00 \\
La Pereza & 2,70 \\
Quebrada Seca & 2,50 \\
Laguna del Río Cuarto (Costa Rica) & 1,38 \\
Esta investigación & 1,30 \\
D & $-0,29$ unidades \\
\hline
\end{tabular}

$\mathrm{D}=(\mathrm{xi}-\overline{\mathrm{X}}) / \overline{\mathrm{X}}$ (Hakanson 1981): desviación del valor medio obtenido en este estudio en relación al valor de la media aritmética. / $D=(x i-\overline{\mathrm{X}}) / \overline{\mathrm{X}}$ (Hakanson 1981): deviation of the mean value obtained in this study in relation to arithmetic mean value.

No concordamos plenamente con lo previsto inicialmente respecto a que la turbidez relativamente alta y la poca penetración de luz 
imperantes en la represa la mayor parte del año hacen deficiente el clima lumínico y la absorción de carbono, ya que aunque hay obvias restricciones lumínicas, el hipolimnio y la profundización de la $\mathrm{D}_{\text {mix }}$ por el patrón de corrientes dentro del cuerpo de agua podrían constituirse en una fuente autóctona de nutrientes que podrían ser cargados a la zona fótica con los concomitantes incrementos de la $\mathrm{P}$, especialmente durante los periodos lluviosos. Esto llevaría además a rechazar el supuesto de que a la represa del embalse no alcanzan a llegar los aportes alóctonos ni los autóctonos y a ratificar la previsión (4) concerniente a que, en los periodos húmedos, cuando disminuyen el clima lumínico y las concentraciones de nutrientes, se presentan los mayores valores de P. No obstante, es necesario recalcar que pesar de la alta concentración de clorofila por unidad de área, comparaciones hechas con otros embalses muestran que la asimilación de carbono es relativamente baja.

Tampoco concordamos con la segunda previsión en cuanto a que la zona de represa tiene un metabolismo heterotrófico ya que, aunque la acumulación de biomasa incrementa la $\mathrm{R}$ dentro del sistema, lo hace con una intensidad levemente pronunciada. Prueba de ello, es que P es más alta que $\mathrm{R}$ la mayor parte del tiempo, lo que implica que el sistema es más autotrófico, comportándose como un sumidero de carbono que tiende a la eutrofia y no como un proveedor de $\mathrm{CO}_{2}$ como se predijo en la tercera hipótesis. Por tanto, y en discordancia con la previsión (5) la zona de represa no está alejada la mayor parte del tiempo de una condición estable, ya que el sistema es capaz de mantenerse en condiciones de estabilidad debido a que por su coeficiente metabólico (R/B) relativamente alto soporta cargas entrópicos o provenientes de sus alrededores.

Respecto al estado trófico, todo apunta a que la represa es eutrófica: la concentración obtenida de B fitoplanctónica $\left(17,1 \pm 11,7\right.$ mgCl.a.m $\left.{ }^{-3}\right)$, los valores medios del coeficiente de asimilación superiores a $200 \mathrm{mgC} \cdot \mathrm{m}^{-3} \cdot \mathrm{d}^{-1}$ para lagos eutróficos confirman esta condición, así como el valor medio de PPB anual (437.3 gC. $\mathrm{m}^{-2} \cdot \mathrm{a}^{-1}$ ) que es mayor que el citado por Margalef (1993) (300 gC.m $\left.\mathrm{m}^{-2} \cdot \mathrm{a}^{-1}\right)$ para sistemas eutróficos.

\section{AGRADECIMIENTOS}

Este trabajo fue realizado usando los datos obtenidos en el proyecto "Caracterización limnológica del embalse Riogrande II, Antioquia, Colombia" (cód. 418) aprobado por el Comité de Investigaciones (CODI) de la Universidad de Antioquia. Agradecemos la importante contribución de un evaluador desconocido al producto final que constituye este escrito.

\section{REFERENCIAS}

Aguirre-Ramírez, N. J., Palacio Baena, J. P., \& Ramírez-Restrepo, J. J. 2007. Características limnológicas del embalse Peñol-Guatapé, Colombia. Revista Ingenierías, Universidad de Medellín, 6(10), 53-66.

Ahlgren, G. 1970. Limnological studies of Lake Norviken, an eutrophicated Swedish lake. II. Phytoplankton and its production. Swiss Journal of Hydrology, 32(2), 353-396. DOI: 10.10 07/BF02502554

Angelini, R., \& Gomes, L. C. 2008. O artesão de ecossistemas: construindo modelos com dados. 1st ed. Maringá, PR: Eduem: p. 173.

Baxter, R. M., Prosser, M. V., Talling, J. F., \& Wood, R. B. 1965. Stratification in tropical African lakes at moderate altitudes $(1,500$ to $2,000 \mathrm{~m})$. Limnology and Oceanography, 10(4), 510-520. DOI: 10.4319/lo.1965.10.4.0510

Beadle, L. C. 1974. The inland waters of tropical Africa: an introduction to tropical limnology. 1st ed. London, UK: Longman Group (Far East): p. 365.

Boynton, W. R., Kemp, W. M., \& Keefe, C. W. 1982. A comparative analysis of nutrients and other factors influencing estuarine phytoplankton production. In: V. S. Kennedy (ed.), Estuarine comparisons. pp. 69-90. New York: Academic Press.

Bustamante Gil, C., Ramírez-Restrepo, J. J., Boltovskoy, A., \& Vallejo, A. 2012. Spatial and temporal change characterization of Ceratium furcoides (Dinophyta) in the equatorial reservoir Riogrande II, Colombia. Acta Limnologica Brasiliensia, 24(2), 207-219. DOI: 10.1590/S217 9-975X2012005000039 
Cole, G. A. 1983. Textbook of limnology. 3rd ed. Saint Louis, MO: Mosby Company: p. 401.

Cole, J. J. 1999. Aquatic microbiology for ecosystem scientists: new and recycled paradigms in ecological microbiology. Ecosystems, 2(3), 215-225. DOI: $10.1007 / \mathrm{s} 100219900069$

Degens, E. T., Deuser, W. G., von Herzen, R. P., Wong, H. K., Wooding, F. B., Jannasch, H. W., \& Kanwisher, J. W. 1971. Lake Kiwu expedition: geophysics, hydrography, sedimentology (Preliminary report). Technical Report No. 71-52; p. 41. Massachusetts: Woods Hole Oceanographic Institution. Retrieved from http://hdl.handle. net/1912/2205

Duarte, C. M., \& Agustí, S. 1998. The $\mathrm{CO}_{2}$ balance of unproductive aquatic ecosystems. Science, 281(5374), 234-236. DOI: 10.1126/science.281.5 374.234

EPM - Empresas Públicas de Medellín. 1994. Proyecto de aprovechamiento múltiple del Río Grande. Declaración de impacto Ambiental. Relato técnico. Empresas Públicas de Medellín, Medellín. p. 210.

Esteves, F. A. 1998. Fundamentos de Limnologia. 2a ed. Rio de Janeiro, RJ: Interciência: p. 602.

Franco Velásquez, J. D. 2011. Modelación de la estructura térmica de un embalse ramificado mediante el análisis de los procesos físicos gobernantes: aplicación al embalse multipropósito Riogrande II. Maestría thesis. Escuela de Geociencias y Medio Ambiente de la Universidad Nacional de Colombia. p. 84.

Franco Velásquez, J. D., Botero, F. M. T., \& GómezGiraldo, A. 2010. Efecto de la posición de la compuerta de captación sobre la estructura térmica de un embalse tropical. XXIV Congreso Latinoamericano de Hidráulica Punta del Este, Uruguay. Obtenido de http://www.bdigital.unal. edu.co/4532/1/DA_265.pdf

Gaarder, T., \& Gran, H. H. 1927. Investigations of the production of plankton in the Oslo Fjord. Rapports et procès-verbaux des réunions, International Council for the Exploration of the Sea, 42. Copenhague: Andr. Fred. Høst \& Fils: p. 52.

Ganf, G. G. 1975. Photosynthetic production and irradiance-photosynthesis relationships of the phytoplankton from a shallow equatorial lake (Lake George, Uganda). Oecologia, 18(3), 165183. DOI: $10.1007 /$ BF00345420
Gaviria, S. 1991. Monitoreo de embalses del sistema de acueducto de Bogotá. Revista Acodal, 147, 29-47.

Gocke, K., Bussing, W., \& Cortés, J. 1990. The anual cycle of primary productivity in Laguna de Río Cuarto, a volcanic lake (maar) in Costa Rica. Revista de Biología Tropical, 38(2B), 387-394.

González, E. J., Ortaz, M., Peñaherrera, C., Montes, E., Matos, M. L., \& Mendoza, J. 2003. Fitoplancton de cinco embalses de Venezuela con diferentes estados tróficos. Limnetica, 22(1-2), 15-35.

Hakanson, L. 1981. A manual of lake morphometry. 1st ed. New York: Springer-Verlag: p. 80.

Harris, G. P.1978. Photosynthesis, productivity and growth: the physiological ecology of phytoplankton. Archiv für Hidrobiologie, 10. Stuttgart: Lubrecht \& Cramer Ltd: p. 171.

Harris, G. P., Haffner, G. D., \& Piccinin, B. B. 1980. Physical variability and phytoplankton communities II. Primary productivity by phytoplankton in a physically variable environment. Archiv für Hydrobiologie, 88, 393-425.

Kalff, J. 1983. Phosphorus limitation in some tropical African lakes. Hydrobiologia, 100(1), 101-112. DOI: 10.1007/BF00027425

Hernández, C., \& Gocke, K. 1990. Productividad primaria en la Ciénaga Grande de Santa Marta, Colombia. Anales del Instituto de Investigaciones Marinas de Punta Betín, 19-20, 101119.

Lemoalle, J. 1969. Premières données sur la production primaire dans la región de Bol (abril-Octobre 1968) (lac Tchad). Cahiers ORSTOM, Série Hydrobiologie, 3(1), 107-119.

Loaiza-Restano, A. M., Ramírez-Restrepo, J. J., Echenique, R. O., Vallejo, A., \& Ortiz, M. L. 2011. Dinámica espacio-temporal de cuatro especies de Staurastrum (Meyen 1829; emend. Ralfs 1848) en un embalse eutrófico colombiano. Oecologia Australis, 15(3), 726-746. DOI: 10.4257/oeco.20 11.1503.22

Mackereth, F. J. H., Heron, J., \& Talling, J. F. 1978. Water analysis: some revised methods for limnologists. Ambleside: Freshwater Biological Association: p. 120.

Margalef, R. 1974. Ecología. Barcelona: Ediciones Omega: p. 951.

Margalef, R. 1983. Limnología. Barcelona: Ediciones Omega: p. 1010. 
Margalef, R. 1993. Teoría de los sistemas ecológicos. 2a ed. Barcelona, ESP: Universidad de Barcelona: p. 290.

Mazo, D., Ramírez-Restrepo, J. J., \& Díaz-C, A. 2015. Caracterización física y química del embalse Riogrande II (Antioquia), Colombia. Actualidades Biológicas, 37(103), 155-168. DOI: 10.17533/udea.acbi.v37n103a04

Melack, J. M., \& Kilham, P. 1974. Photosynthetic rates of phytoplankton in east African alkaline saline lakes. Limnology and Oceanography, 19(5), 743-755. DOI: 10.4319/lo.1974.19.5.0743

Montoya, Y., \& Ramírez-Restrepo, J. J. 2007. Flujos de mineralización en el embalse tropical Riogrande II (Antioquia, Colombia). Limnetica, 26(1), 39-51.

Naundorf, G. 1990. Caracterización de la comunidad fitoplanctónica y determinación de la productividad primaria del embalse "La Salvajina” y su área de influencia. Revista Asociación Colombiana de Ciencias Biológicas, 7, 154-163.

Nixdorf, B., \& Deneke, R. 1997. Why 'very shallow' lakes are more successful opposing reduced nutrient loads. Hydrobiologia, 342/343(0), 269284. DOI: 10.1023/A:1017012012099

Odum, E. P. 1969. The strategy of ecosystem development. Science, 164(3877), 262-270. DOI: 10.1126/science.164.3877.262

Odum, E. P. 1972. Ecología. 3a ed. Rio de Janeiro, RJ: Interamericana: p. 639.

Powell, T. M. 1989. Physical and biological scales of variability in lakes, estuaries, and the coastal ocean. In: J. Roughgarden, R. M. May \& S. A. Levin (Eds.), Perspectives in ecological theory. pp. 157-180. New Jersey: Princeton University Press.

Porras-Zapata, P., Betancourt-Ángel, A., MolinaArredondo, A. M., Lopera-Pérez, J. D., \& Agudelo-García, R. A. 1997. El recurso agua como elemento ordenador del territorio: zona de influencia del embalse Riogrande II. Colombia: Universidad Nacional de Colombia: p. 102.

Ramírez-Restrepo, J. J., Arcila, L. M. C., \& Sepúlveda, A. S. C. 2015. Cuantificación de los componentes que afectan el coeficiente de atenuación vertical para irradiancia descendente en el embalse Riogrande II (Colombia). Revista de la Academia Colombiana de Ciencias
Exactas Físicas y Naturales, 39(151), 228-238. DOI: $10.18257 /$ raccefyn. 150

Reynolds, C. S. 2006. Ecology of Phytoplankton. Ecology, biodiversity and conservation. 1st ed. Cambridge, UK: Cambridge University Press: $p$. 552.

Roldán Pérez, G., \& Ramírez Restrepo, J. J. 2008. Fundamentos de limnología neotropical. 2a ed. Medellín, CO: Editorial Universidad de Antioquia: p. 440.

Sandoval Rojo, L. C., Flores Verdugo, F. J., Zaragoza Araujo, U., Day J. W. Jr, \& Estrada Mercado, A. 1988. Phytoplankton productivity in the Barra de Navidad coastal lagoon on the Pacific coast of Mexico. Revue d'Hydrobiologie Tropicale, 21(2), 101-108.

Sartory, D. P., \& Grobbelaar, J. U. 1984. Extraction of chlorophyll $a$ from freshwater phytoplankton for spectrophotometric analysis. Hydrobiologia, 114(3), 177-187. DOI: 10.1007/BF00031869

Schindler, D. W. 1978. Factors regulating phytoplankton production and standing crop in the world's freshwaters. Limnology and Oceanography, 23(3), 478-486. DOI: 10.4319/ lo.1978.23.3.0478

Steemann Nielsen, E. 1952. The use of radioactive Carbon $\left({ }^{14} \mathrm{C}\right)$ for measuring organic production in the sea. ICES Journal of Marine Science, 18(2). Journal du Conseil / Conseil Permanent International pour l'Exploration de la Mer18, 117-140. DOI: 10.1093/icesjms/18.2.117

Straškraba, M. 1999. Retention time as a key variable of reservoir limnology. In: J. G. Tundisi \& M. Straškraba (Eds.), Theoretical reservoir ecology and its applications. pp. 385-410. São Carlos: International Institute of Ecology, Brazilian Academy of Science and Backhuys Publishers.

Talling, J. F. 1957. The phytoplankton population as a compound photosynthetic system. New Phytologist, 56(2), 133-149. DOI: 10.1111/j.146 9-8137.1957.tb06962.x

Talling, J. F. 1965. The photosynthetic activity of phytoplankton in East African lakes. Internationale Revue der gesamten Hydrobiologie und Hydrographie, 50(1), 1-32. DOI: 10.1002/iroh.19 650500102

Talling, J. F. 1970. Generalized and specialized features of phytoplankton as a form of photosynthetic cover. In: International Biolo- 
gical Programme, Section of Production Processes \& the Czechoslovak Academy of Sciences (Eds.), Prediction and measurement of photosynthethic productivity: proceedings of the IBP/PP technical meeting, Trebon. pp. 431445. Wageningen: PUDOC.

Talling, J. F. 1971. The underwater light climate as a controlling factor in the production ecology of freshwater phytoplankton. SIL Communications, 1953-1996. Internationale Vereinigung für Theoretische und Angewandte Limnologie: Mitteilungen, 19(1), 214-243. DOI: 10.1080/053 84680.1971.11903932

Talling, J. F. 1976. The depletion of Carbon dioxide from lake water by phytoplankton. Journal of Ecology, 64(1), 79-121. DOI: 10.2307/2258685

Tundisi, J. G. 1983. A review of basic ecological processes interacting with production and standing-stock of phytoplankton in lakes and reservoirs in Brazil. Hydrobiologia, 100(1), 223243. DOI: $10.1007 / \mathrm{BF} 00027431$

Tundisi, J. G., \& Tundisi, T. M. 2008. Limnologia. la ed. São Paulo, SP: Oficina de Textos: p. 632. Vincent, W. F. 1990. The dynamic coupling between photosynthesis and light in the phytoplankton environment.

SIL Proceedings, 1922-2010. Internationale Vereinigung für Theoretische und Angewandte Limnologie: Verhandlungen, 24(1), 25-37. DOI: 10.1080/03680770.1989.11898688

Westlake, D. F., Adams, M. S., Bindloss, M. E., Ganf, G. G., Gerloff, G. C., Hammer, U. T., Javornicky, P., Koonce, J. F., Marker, A. F. H., McCracken, M. D., Moss, B., Nauwerck, A., Pyrina, I. L., Steel, J. A. P., Tilzer, M., \& Walters, C. J. 1980. Primary production. In: E. D. Le Cren \& R. H. LoweMcConnell (Eds.), The functioning of freshwater ecosystems. pp. 141-246. Cambridge: Cambridge University Press.

Presentado en: 23 de febrero de 2014 Aceptado en: 24 de junio de 2014 Editores de Área: Renato Crouzeilles y Natalie Olifiers 